\section{Unusual, but important, peri- and extra- articular manifestations of rheumatoid arthritis: a pictorial essay}

\author{
Ji Young Suh, Sun-Young Park, Sung Hye Koh, In Jae Lee, Kwanseop Lee \\ Department of Radiology, Hallym University Sacred Heart Hospital, Hallym University College \\ of Medicine, Anyang, Korea
}

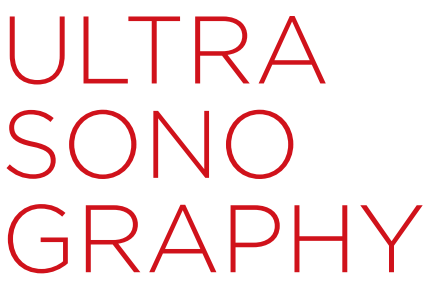

\section{PICTORIAL ESSAY}

https://doi.org/10.14366/usg.20161 pISSN: 2288-5919 - elSSN: 2288-5943 Ultrasonography 2021;40:602-616

Received: October 7, 2020

Revised: January 30, 2021

Accepted: February 2, 2021

Correspondence to:

Sun-Young Park, MD, Department of Radiology, Hallym University Sacred Heart Hospital, Hallym University College of Medicine, 22 Gwanpyeongro 170beon-gil, Dongan-gu, Anyang 14068, Korea

Tel. +82-31-380-5985

Fax. $+82-31-380-3878$

E-mail: sunypark83@naver.com

\title{
Introduction
}

Rheumatoid arthritis (RA) is a systemic, chronic inflammatory disorder of unknown etiology. RA mainly affects the joints; however, it can also have multiple peri- and extra-articular manifestations. The fundamental target area of RA is the synovium, which causes synovitis when antigens present in the synovial membrane elicit a cellular immune response. In addition, RA affects the synovium-lined tendon sheaths, bursae, and entheses where ligaments and tendons attach to bone. Ultrasonography (US) is a non-invasive, cost-effective diagnostic tool for evaluating synovitis that can easily be applied in outpatient clinics. When a patient with RA undergoes a US examination, radiologists tend to focus on synovitis. Furthermore, peri- or extra-articular manifestations are difficult to detect at initial presentation without specific medical history or clinical information. Therefore, radiologists should be knowledgeable of the various imaging characteristics of the broad disease spectrum to enable a correct and early diagnosis of RA. Furthermore, these various and unusual imaging findings could assist rheumatologists in making appropriate therapeutic decisions for patients with RA.

Herein, we describe the broad spectrum of ultrasonographic characteristics of peri- and extraarticular involvement to assist in the accurate diagnosis of RA.

\section{Peri-articular Manifestations}

\section{Tendon}

RA affects the synovial lining of the tendon sheath, which causes tenosynovitis. Tenosynovitis is an
This is an Open Access article distributed under the terms of the Creative Commons Attribution NonCommercial License (http://creativecommons.org/ licenses/by-nc/4.0/) which permits unrestricted noncommercial use, distribution, and reproduction in any medium, provided the original work is properly cited.

Copyright (C) 2021 Korean Society of Ultrasound in Medicine (KSUM)

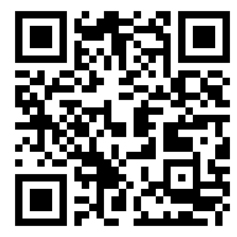

How to cite this article: Suh JY, Park SY, Koh SH, Lee IJ, Lee K. Unusual, but important, peri- and extra-articular manifestations of rheumatoid arthritis: a pictorial essay. Ultrasonography. 2021 0ct;40(4):602-616. 
early phenomenon occurring in patients at risk of RA, and is a predictive factor of RA development [1]. The reported prevalence of tendon involvement in patients with RA is considerably high, ranging from $12.2 \%$ to $50.0 \%$ for hand and ankle tendons [2]. The histopathological mechanism of RA tenosynovitis is similar to that of joint synovitis, including hyperplasia of the synovial lining and infiltration of inflammatory cells and mediators. On US, tenosynovitis presents as hypoechoic or anechoic thickening of the tendon sheath, often accompanied by surrounding tenosynovial fluid within the sheath [3-6]. Color Doppler US can be used to examine hyperemia associated with active inflammation or chronic disease exacerbation
[3-6].

Although any tendon may be affected, extensor tendon sheaths at the level of the wrist, from compartments I to $\mathrm{VI}$, are more frequently affected than those of the flexors (Fig. 1) [4,6]. The extensor carpi ulnaris tendon is a common site affected by RA (Fig. 2) [3,6]. At the level of the ankle, the tibialis posterior (Fig. 3), flexor digitorum longus, flexor hallucis longus, and peroneal tendons are more frequently involved than are the extensors [4]. Moreover, isolated tenosynovitis without joint involvement can occur.

In the chronic stage, partial or complete tendon rupture may result from the weakening of the tendon sheath caused by infiltrative

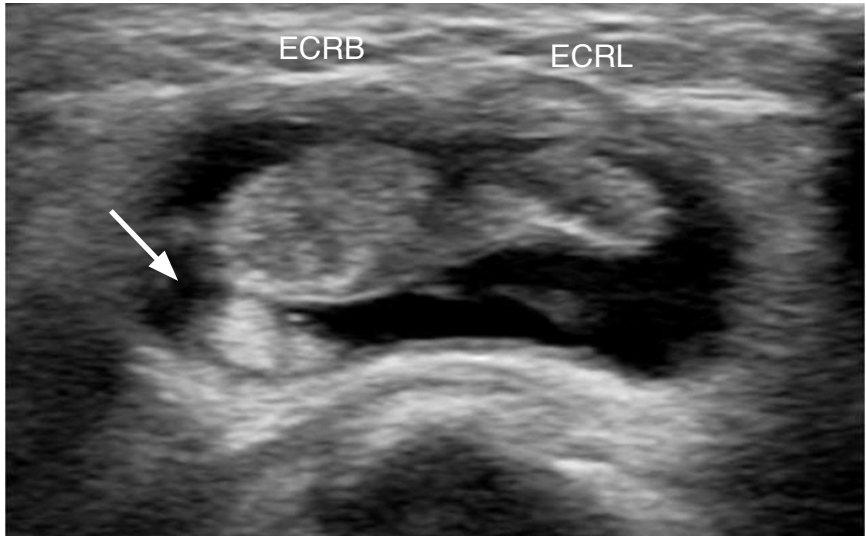

A

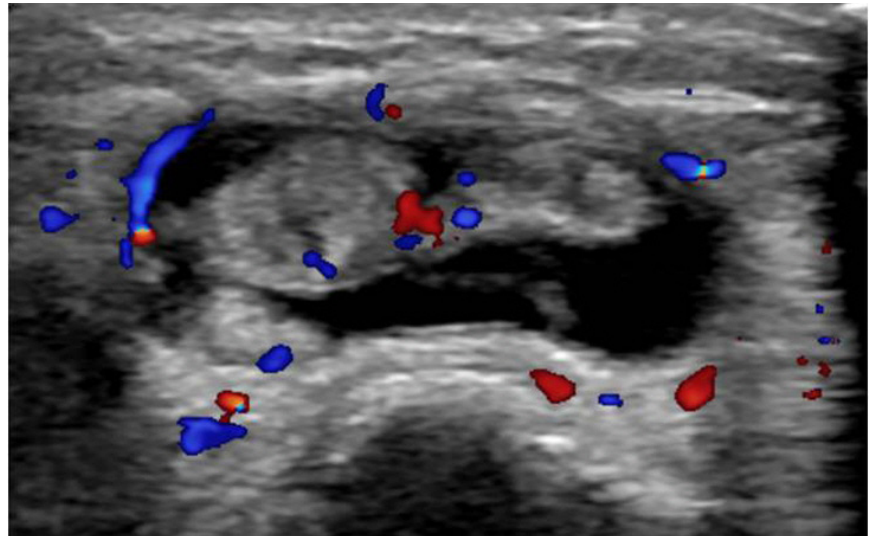

B

Fig. 1. Second extensor compartment tenosynovitis of the wrist in a 45-year-old woman.

A patient who had been on medication for seropositive rheumatoid arthritis complained of pain in the right wrist. A. Grayscale ultrasonography shows a loculated fluid collection along with the second extensor compartment (extensor carpi radialis brevis [ECRB] and extensor carpi radialis longus [ECRL]) with internal synovial proliferation (arrow) on an axial scan. B. Color Doppler ultrasonography shows hyperemia in the lesion. These findings represent tenosynovitis in the second extensor compartment (ECRL, ECRB) of the right wrist.

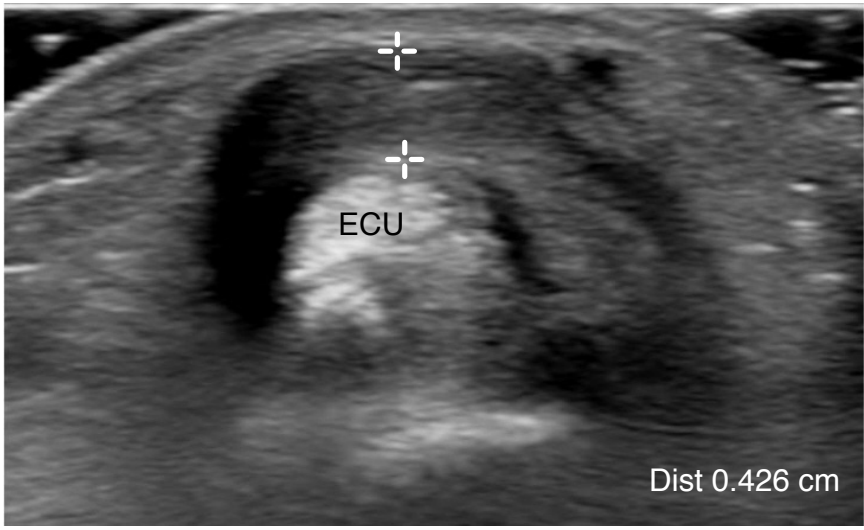

A

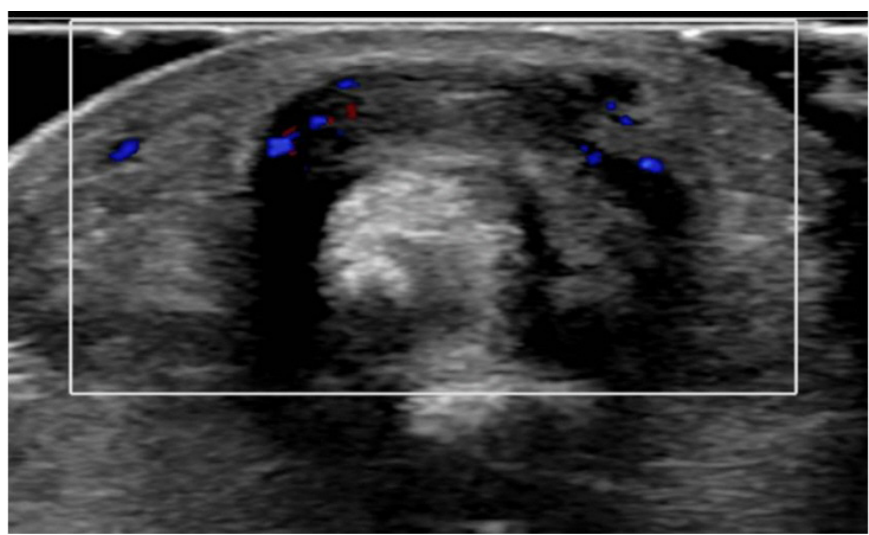

B

Fig. 2. Extensor carpi ulnaris (ECU) tenosynovitis in a 45-year-old woman.

A patient with severe seropositive rheumatoid arthritis complained of pain and swelling of the left wrist. Neither synovial thickening nor joint effusion at the left wrist was found (not seen in the figures). A. Grayscale ultrasonography shows diffuse thickening of the ECU tendon sheath. B. Color Doppler ultrasonography shows increased color flow of the thickened tendon sheath and surrounding soft tissue. 


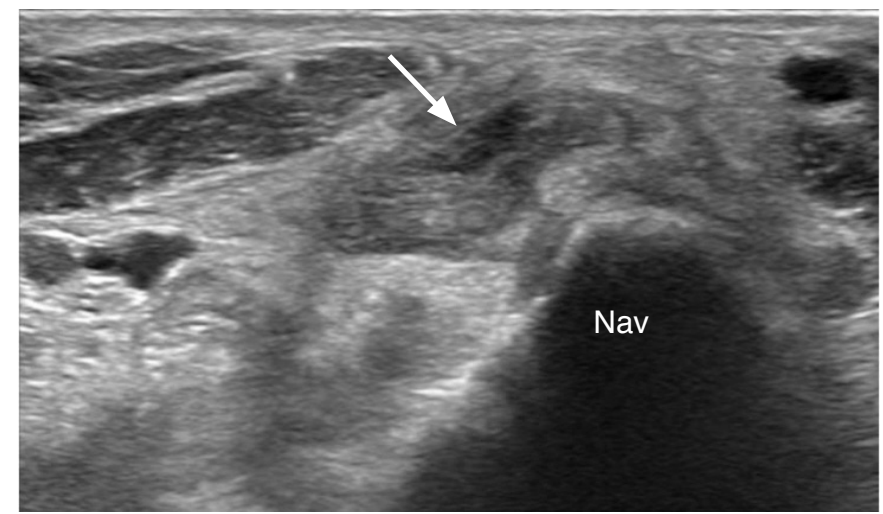

A

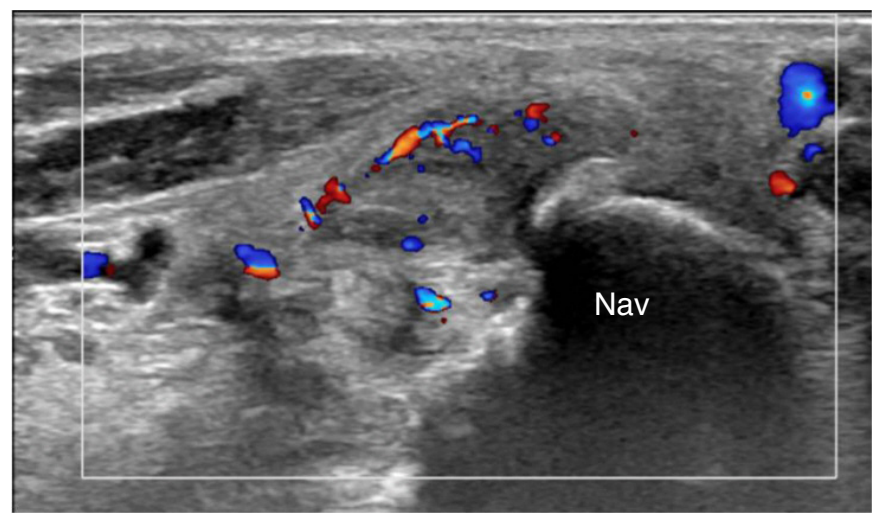

B

Fig. 3. Tenosynovitis in the tibialis posterior tendon in a 48-year-old woman.

The patient complained of pain and swelling in the medial aspect of the left ankle and foot. She had been on medication for rheumatoid arthritis for several years. Neither joint space narrowing nor bony erosion in the left foot and ankle was found on plain radiography (not seen on the figures). A. On grayscale ultrasonography, there is a markedly swollen tibialis posterior tendon at the navicular (Nav) attachment site with surrounding soft-tissue edema. There is a focal hypoechoic lesion into the swollen tendon, suggesting a partial tear (arrow). B. Color Doppler ultrasonography shows increased vascularity of the tendon. This is a case of isolated tenosynovitis of the tibialis posterior tendon without joint involvement of rheumatoid arthritis.

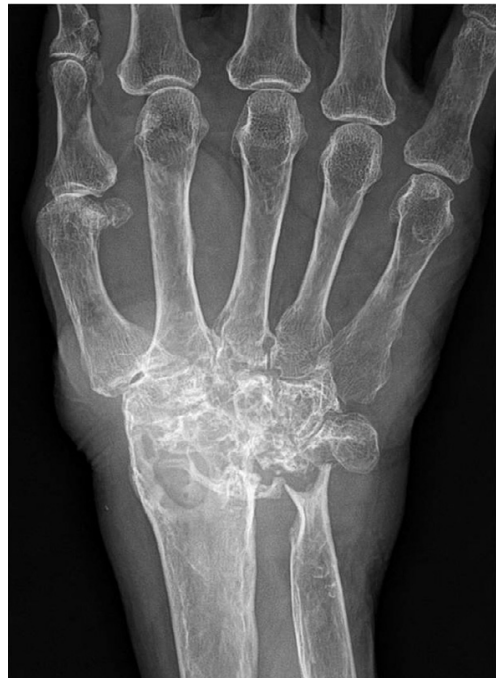

A

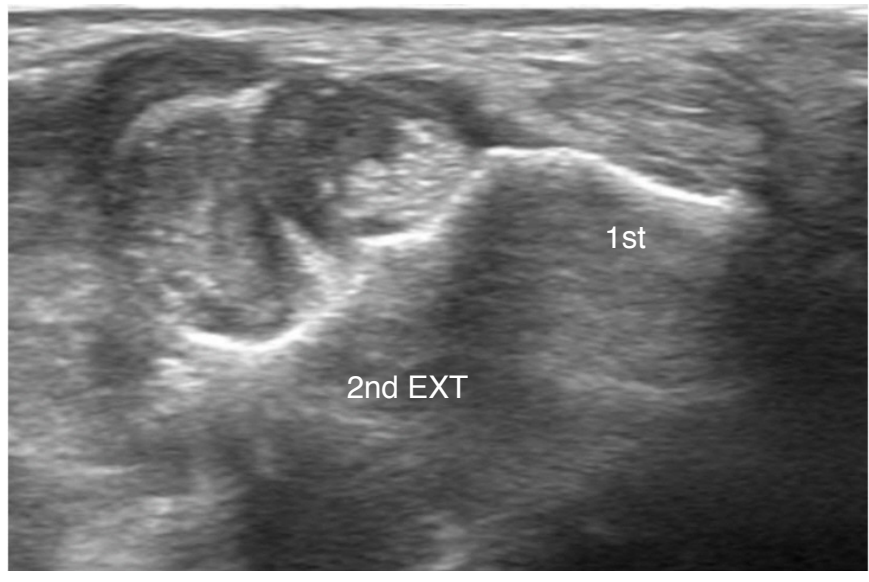

B

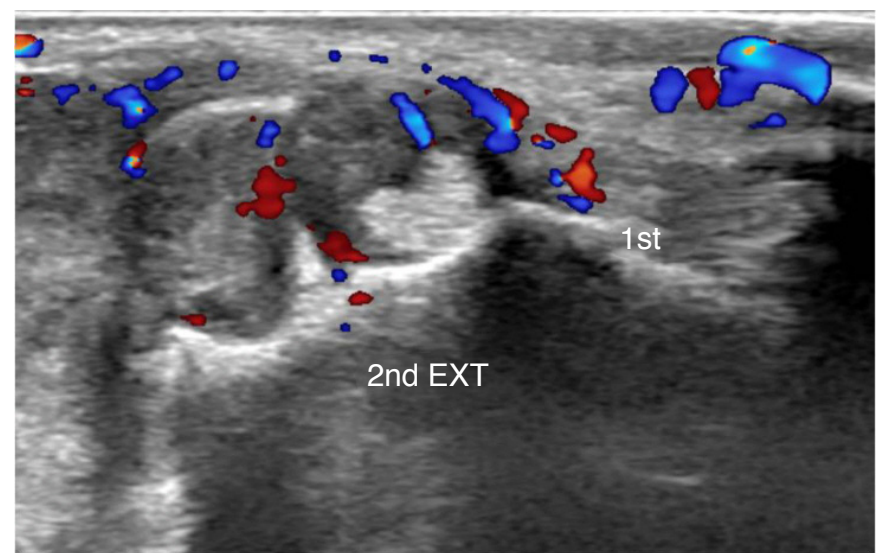

C

Fig. 4. Chronic extensor compartment tenosynovitis of the wrist in a 75-year-old woman.

Eight years ago, the patient presented to the hospital for right wrist pain and was diagnosed with seronegative rheumatoid arthritis. At that time, mild subchondral bone erosion of carpal bones and tenosynovitis of the flexor tendons were found on magnetic resonance imaging (not seen on the figures). However, there was no rheumatoid arthritis involvement in the extensor compartment of the wrist. For several years, she had suffered from chronic wrist pain. A. Plain radiography after 8 years shows severe peri-articular bone erosions with deformity of the wrist joint. B. Ultrasonography on the same day shows diffuse thickening of the tendon sheaths of the first and second extensor (EXT) compartments and adjacent bony erosion. C. There is markedly increased vascularity of the lesion on color Doppler ultrasonography. 
synovitis or from friction caused by tendon movement across the irregularly eroded bone surface (Fig. 4) [7,8]. Caput ulnae syndrome is characterized by end-stage destruction of the distal radioulnar joint caused by RA. It results in dorsal dislocation of the distal ulna, supination of the carpus, and volar subluxation of the extensor carpi ulnaris tendon. Eventually, the ulnar-side extensor tendons directly overlie the ulna prominence, which leads to tendon rupture, particularly on the ulnar side (Fig. 5).

In the wrist, the differential diagnoses of RA tenosynovitis include other infectious or inflammatory conditions such as tuberculosis (TB), psoriatic arthritis, and overuse tendon injury. RA is difficult to

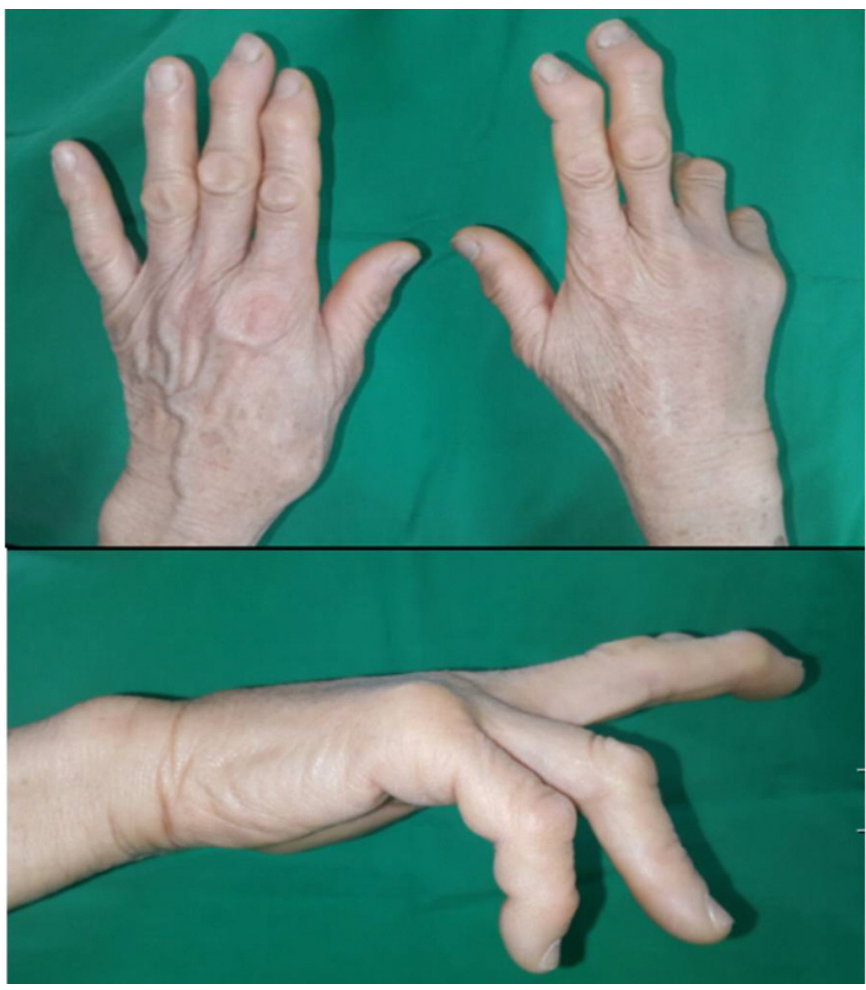

A

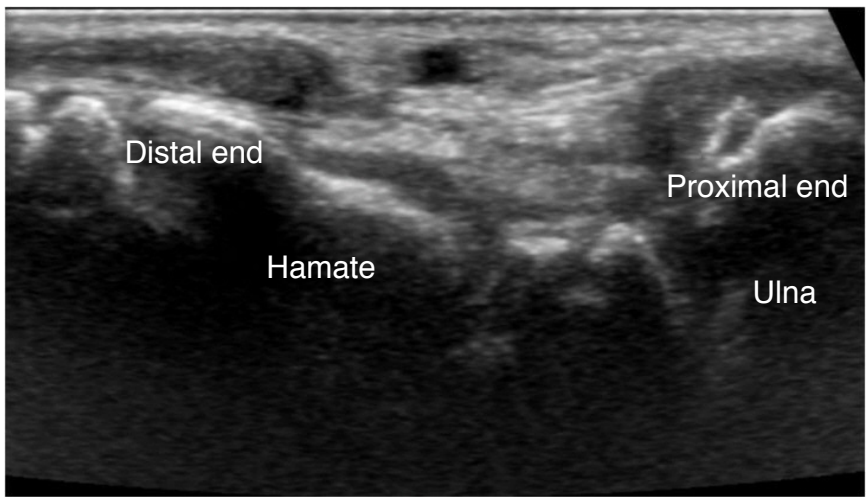

C distinguish because other diseases can have similar features on US; however, a few points can be helpful for the differential diagnosis. TB tenosynovitis may be included in the differential diagnosis due to the chronic course of disease with similar clinical characteristics to RA. Similar findings, such as tendon sheath fluid with synovial proliferation and tendon sheath thickening, can be seen on US. However, TB tenosynovitis selectively targets the wrist and the volar aspect of the flexor tendon [9]. A lack of other rheumatoid features would make TB tenosynovitis more likely. In addition to TB infection, seronegative spondyloarthropathy may have similar imaging findings to RA. Spondyloarthropathy is mainly differentiated from RA by the

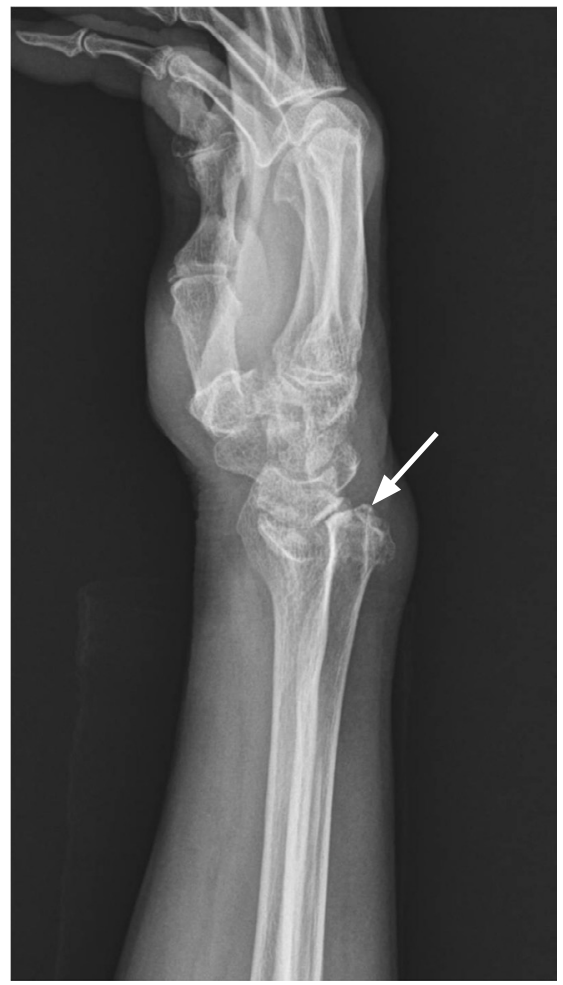

B

Fig. 5. Caput ulnae syndrome in a 76-year-old woman.

A. A patient with longstanding medication for rheumatoid arthritis complained of finger drop involving the fourth and fifth phalanges with a palpable lesion on the dorsum of the right ulna. B. On a plain radiograph, there is dorsal subluxation of the ulna (arrow) with arthritis at the distal radioulnar joint. C. On ultrasonography, the extensor digitorum communis and extensor digiti minimi are ruptured. The proximal end of the ruptured tendon is located at the level of the ulnar end and the distal end is located at the level of the hamate. This is a case of caput ulnae syndrome and ruptured extensor tendon of the wrist in a patient with rheumatoid arthritis. 


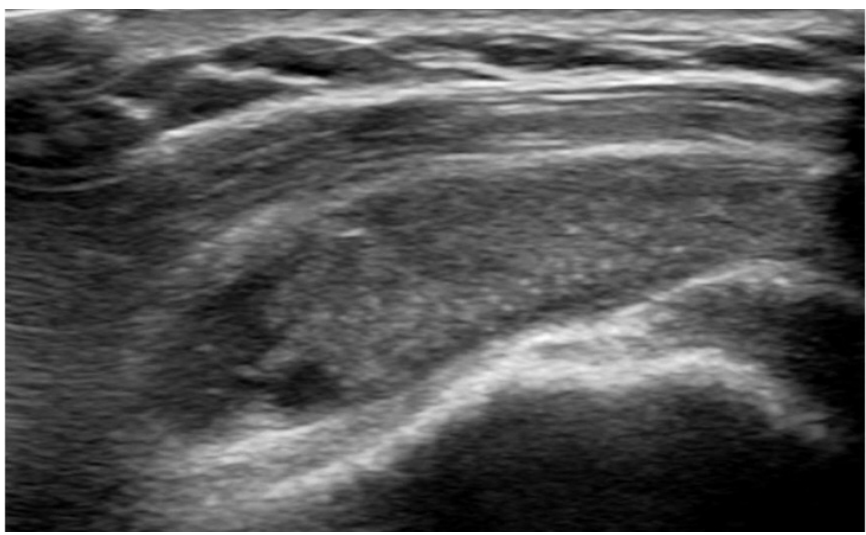

A

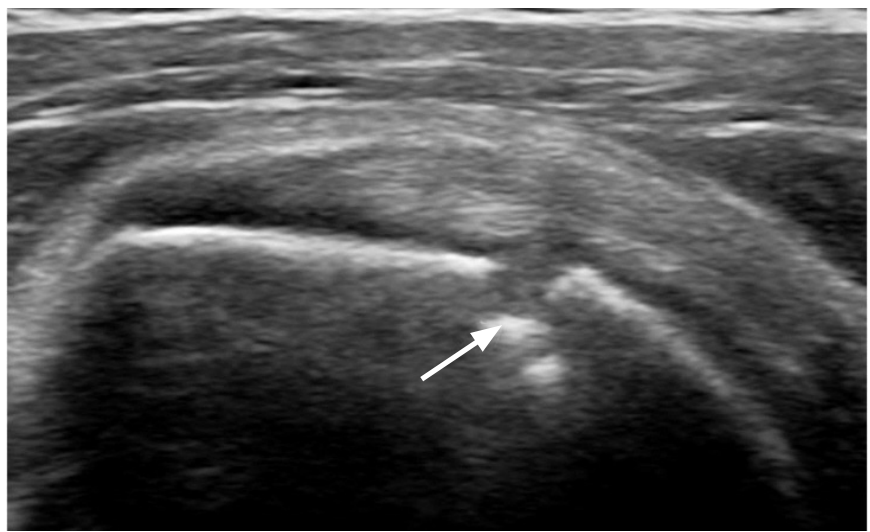

C

Fig. 6. Subacromial-subdeltoid bursitis in a 40-year-old woman.

A patient on medication for rheumatoid arthritis complained of pain and swelling of the right shoulder joint. A. Ultrasonography shows diffuse synovial thickening, synovial proliferation, and a small amount of effusion at the subacromial-subdeltoid bursa, suggesting subacromial-subdeltoid bursitis. B. On color Doppler ultrasonography, the vascularity is increased over the subacromial-subdeltoid bursa. C. There is focal bone erosion at the lesser tuberosity of the humeral head (arrow). D. There is tendinosis of the anterior aspect of the supraspinatus tendon with underlying bone erosion at the greater tuberosity (arrow). These are findings of rheumatoid arthritis involving the shoulder.

involvement of non-synovial articular and peri-articular structures. Psoriatic tenosynovitis more commonly involves the flexor tendon of finger in the distal interphalangeal joints rather than the wrist, and entheseal involvement of the fingers, psoriasis, nail changes, and sausage digit may help distinguish it from rheumatoid tenosynovitis [4]. It is difficult to differentiate the early inflammatory features of RA from overuse tenosynovitis, but RA coexists with synovitis in most cases. Additionally, RA tenosynovitis accompanies other RArelated findings such as bone erosion or joint subluxation in the chronic stage $[4,6]$.

\section{Bursa}

RA involves the synovial membrane in the bursae. It can occur in any bursa in the body, particularly the subacromial-subdeltoid,

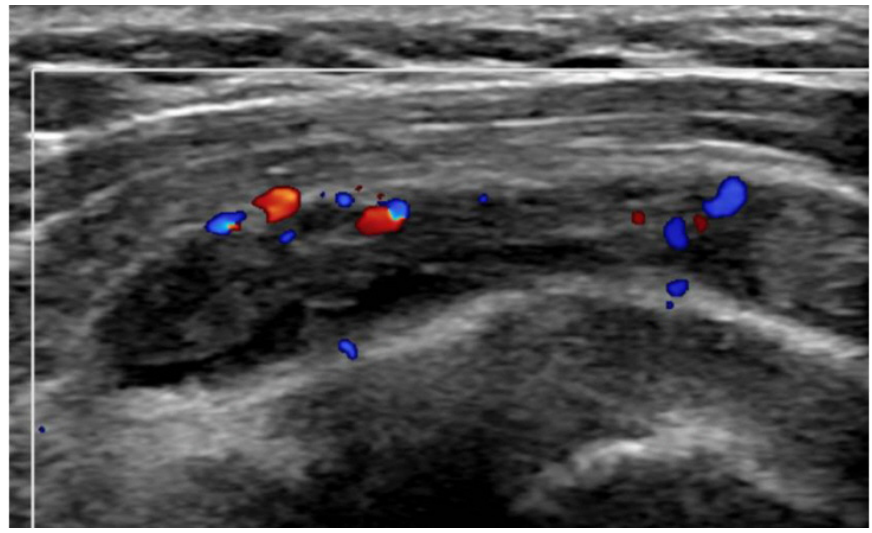

B

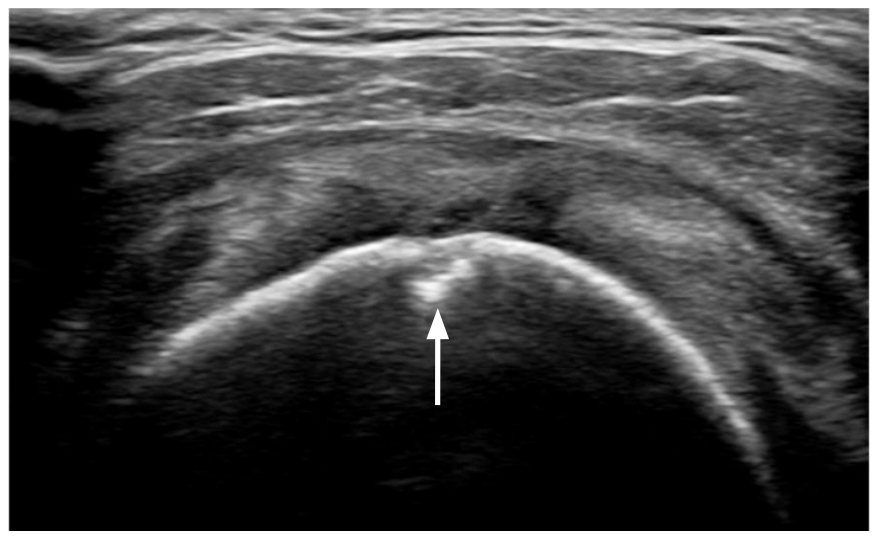

D 


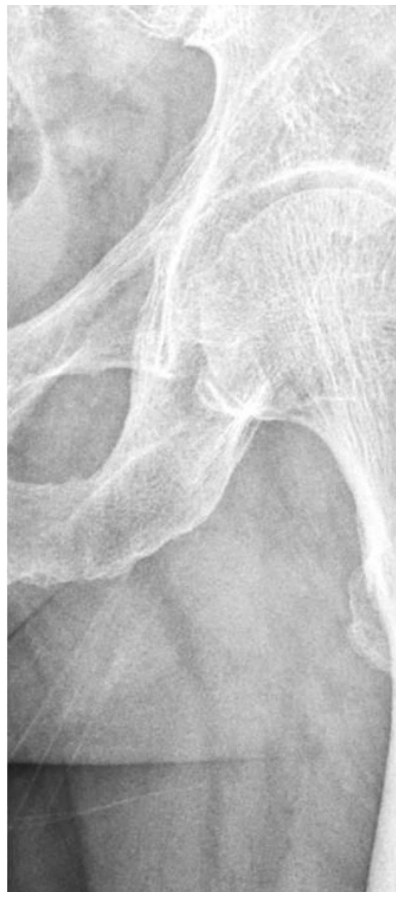

A

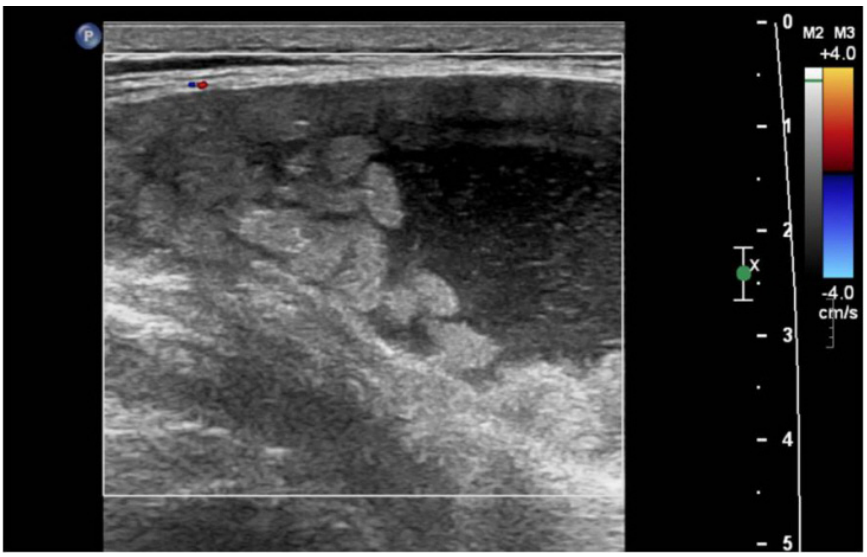

C

characterized by fluid-filled sacs lacking synovial lining. Adventitial bursae are located in the submetatarsal pad, and are considered to be mechanically derived, where the friction or weight-bearing forces result in collagen degradation and concomitant localized fluid [11]. On US, hypertrophied intermetatarsal bursae manifest as well-defined fluid collections with a hypoechoic zone usually bulging $>1 \mathrm{~mm}$ under the metatarsal heads (Fig. 8). In contrast, adventitial bursae present as anechoic or heterogeneous fluid collections within the submetatarsal fat pad (Fig. 9) [11]. Forefoot bursae may reduce the mobility of the foot by causing foot pain and dysfunction. Therefore, it is crucial to look not only for synovitis and tenosynovitis, but also for bursitis, in patients with RA to make an

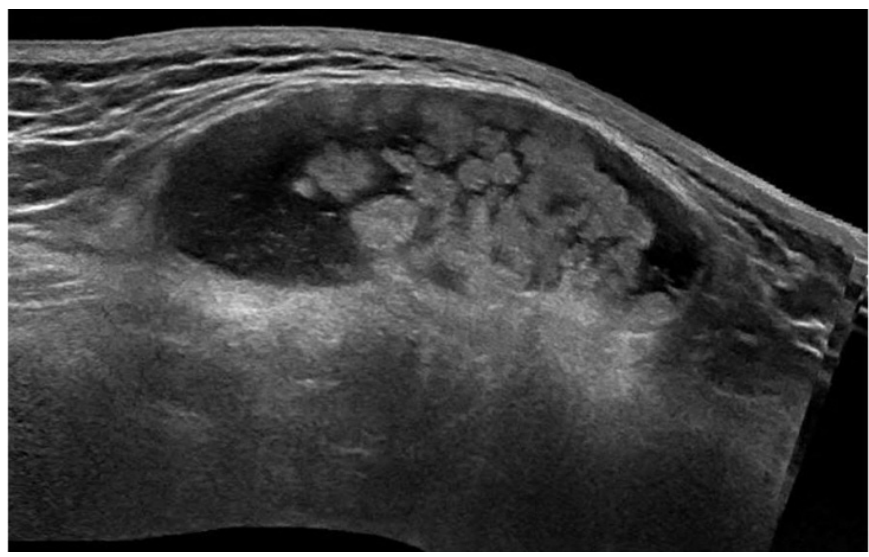

B

Fig. 7. Greater trochanteric bursitis in a 67-year-old woman.

A patient with longstanding seropositive rheumatoid arthritis complained of left hip pain. A. There is no joint space narrowing or deformity on plain radiography, but an oval contour bulge is located at the lateral aspect of greater trochanter of the left femur (arrows). On grayscale ultrasonography, there is no evidence of synovitis in the left hip joint (not seen in the figures). B. Ultrasonography shows a $7-\mathrm{cm}$ fluid collection with internal massive proliferation of rice bodies, over the greater trochanter and lateral to the posterior aspect of the left hip. C. Minimal vascularity is shown on color Doppler ultrasonography, meaning that it is in the chronic stage, rather than an acute state. This is a case of trochanteric bursitis in a patient with rheumatoid arthritis.

accurate diagnosis and to optimize patient care.

\section{Ligament}

An enthesis is the insertion site of a tendon, ligament, or articular capsule into the bone. Entheseal inflammation is often asymptomatic and may be overlooked during a clinical examination. Extra-articular enthesitis is common in spondyloarthropathy; however, it can also occur in RA. On US, enthesitis appears as hypoechoic (loss of normal fibrillar architecture) and/or thickened tendons or ligaments at their bony attachment site, which may present peri-entheseal hyperemia upon a Doppler examination during the acute phase. It may be associated with bone erosion, bursitis, and enthesophytes. 


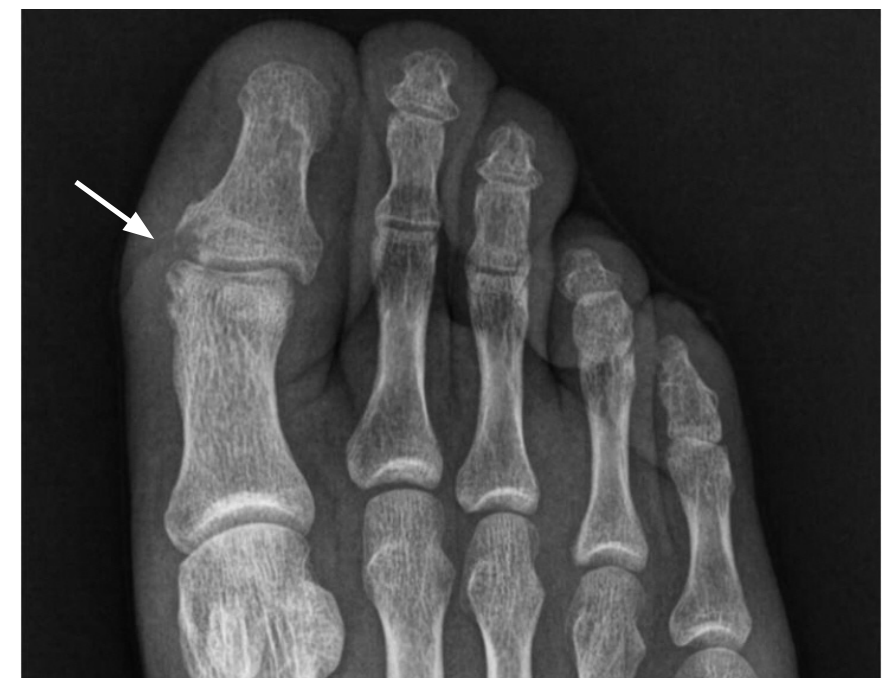

A

Fig. 8. Intermetatarsal bursitis in a 33-year-old man.

This patient had right forefoot pain. He was a rheumatoid arthritis patient with positive anti-cyclic citrullinated peptide findings and had a remarkable family history, as his mother had been on medication for severe rheumatoid arthritis. A. A plain radiograph shows bone erosion in the left first toe (arrow). B. Ultrasonography over the sole side of the left foot on the same day indicates a $1-\mathrm{cm}$ ill-defined heterogeneous hypoechoic lesion with a thick peripheral wall in the third web space. C. Increased vascularity is shown on color Doppler ultrasonography. This is a case of intermetatarsal bursitis in the third web space of the right foot in a rheumatoid arthritis patient.

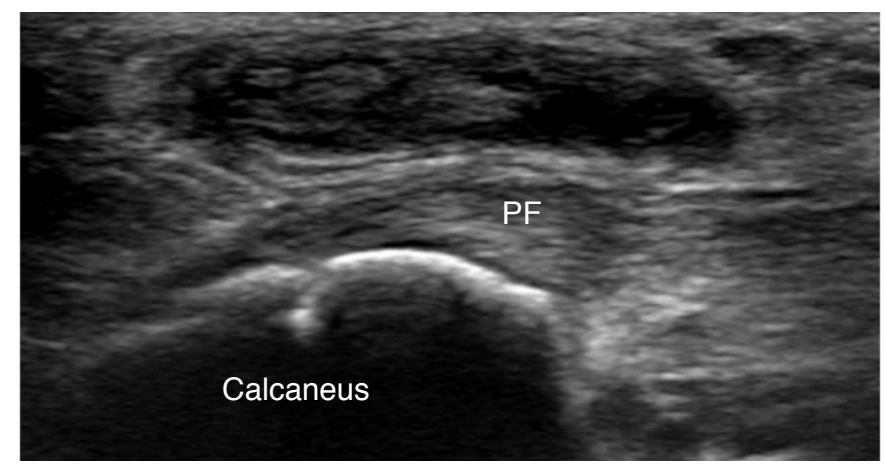

A

Fig. 9. Adventitious bursitis in a 48-year-old woman.

A patient on longstanding medication for rheumatoid arthritis complained of pain in the left heel. A. Grayscale ultrasonography shows a 2.5-cm hypoechoic lesion with a thick peripheral echogenic wall below the plantar fascia (PF). B. Color Doppler ultrasonography shows hyperemia over this lesion. These findings suggest adventitious plantar bursitis of the heel pad.

During the inactive or chronic stage, US shows tendon or ligament thickening, prominent enthesophytes, intratendinous calcification, and bone erosion. McGonagle et al. [12] proposed the concept of the synovio-entheseal complex, where primary entheseal damage may occur from inflammatory reactions at adjacent synovial tissues.

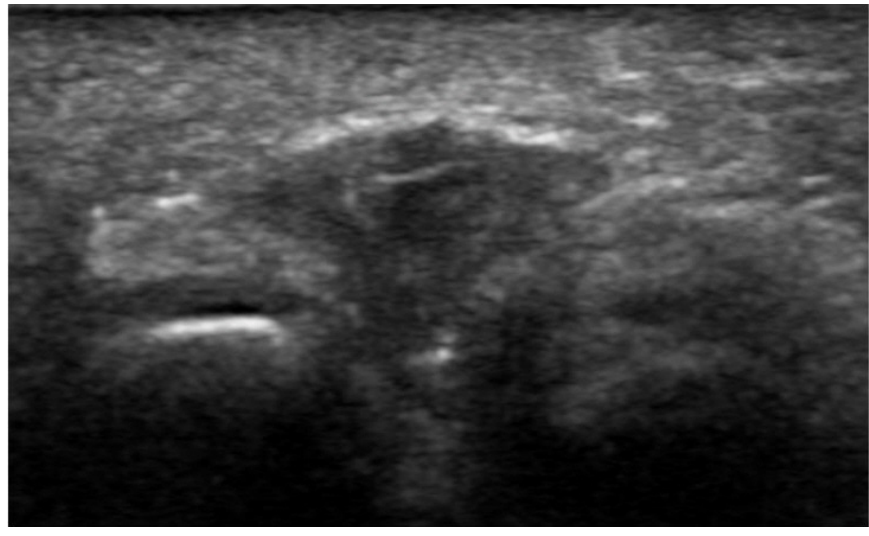

B

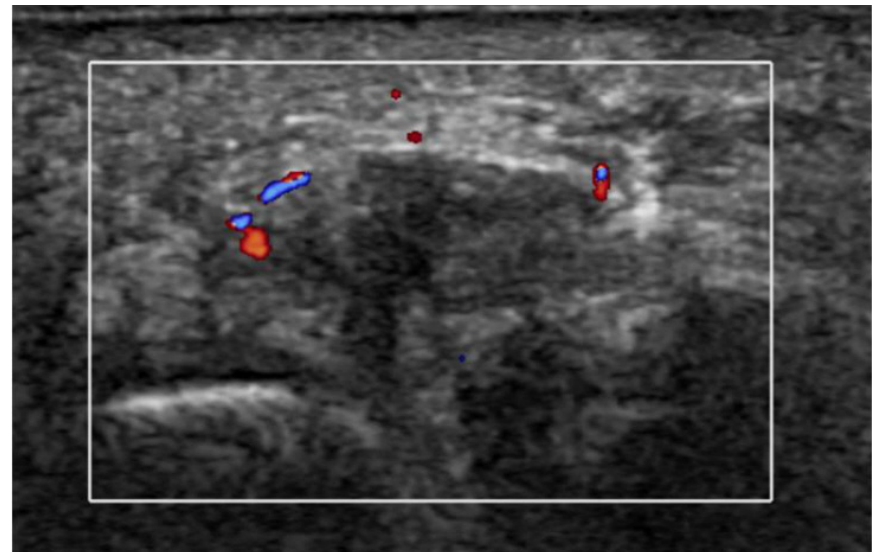

C

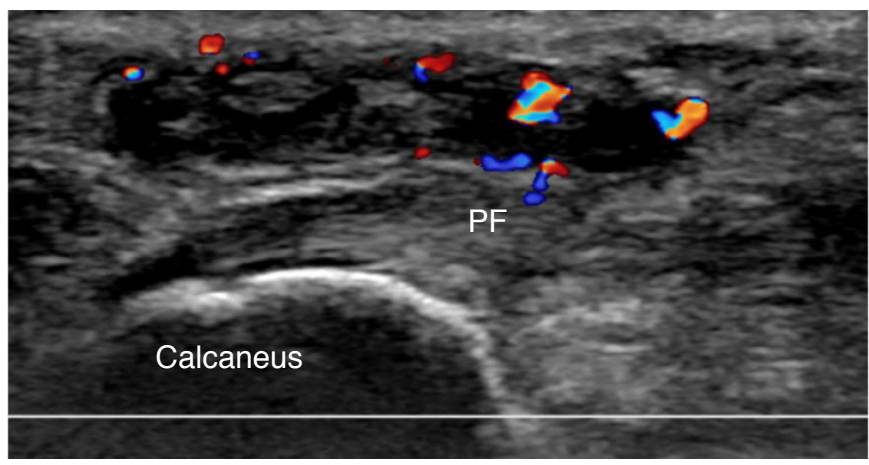

B 
in spondyloarthropathy than in RA, it still occurs in the symptomatic ankles of $22 \%$ of patients with early RA (Fig. 10) [13]. Suzuki et al. [14] recently reported that retrocalcaneal bursitis could be preceded or accompanied by Achilles tendon enthesitis and suggested that synovio-entheseal complex inflammation in RA arises from synovial tissues, supporting the concept proposed by McGonagle et al. [12].

\section{Extra-articular Manifestations}

\section{Subcutaneous Tissue}

Rheumatoid nodules are the most common extra-articular manifestations of RA and are frequent in longstanding RA, with an approximate occurrence of $20 \%-30 \%$ in patients with seropositive RA [8]. However, the pathogenesis of rheumatoid nodules remains unclear. Rheumatoid nodules are common at sites with mechanical irritation and pressure, including the olecranon region often associated with the olecranon bursa (Fig. 11), forearm, finger joints, and, rarely, foot joints (Fig. 12). Nodules are usually asymptomatic, palpable, firm, and easily movable subcutaneous lesions that can become painful when irritated. Mature rheumatoid nodules are composed of inner central necrosis, middle palisading fibroblasts, and an outer collagenous capsule with perivascular infiltration of chronic inflammatory cells [8]. On US, nodules usually appear as lobulated heterogeneous hypoechoic, and non-erosive compressible masses close to the bone surface. Some nodules present as central demarcated hypoechoic areas, which correspond to the central necrotic portion [15].

A common differential includes tophi in gout. Gout nodules mostly appear as heterogeneous masses, and occasionally have calcifications that appear as hyperechoic lesions with acoustic shadowing. Cortical bone erosion can be seen adjacent to the bone surface [15]. Rheumatoid nodules rarely calcify, which is a diagnostic feature that distinguishes them from gout tophi. Additionally, rheumatoid nodules rarely cause subjacent bone erosion [1]

Panniculitis is an acute, self-limiting disorder involving one or more tender nodules. It is very rare, but can be found in RA, similar to erythema nodosum, and may precede or occur concurrently with RA symptoms or appear late in the disease course (Fig. 13) [16].

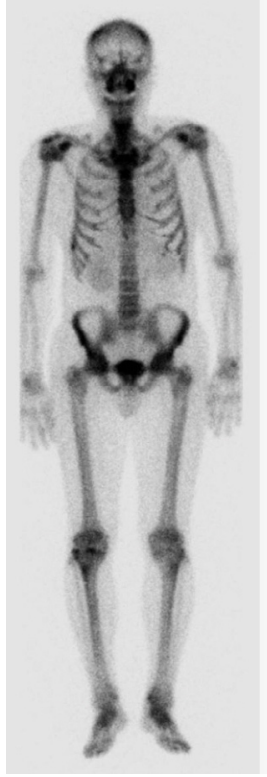

Anterior

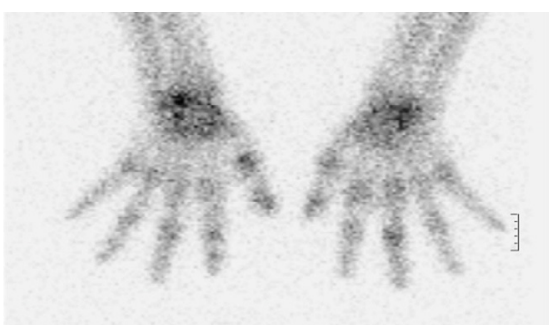

LT

RT

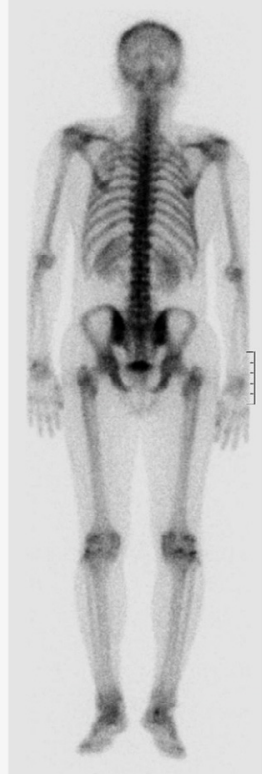

Posterior

A

e-ultrasonography.org

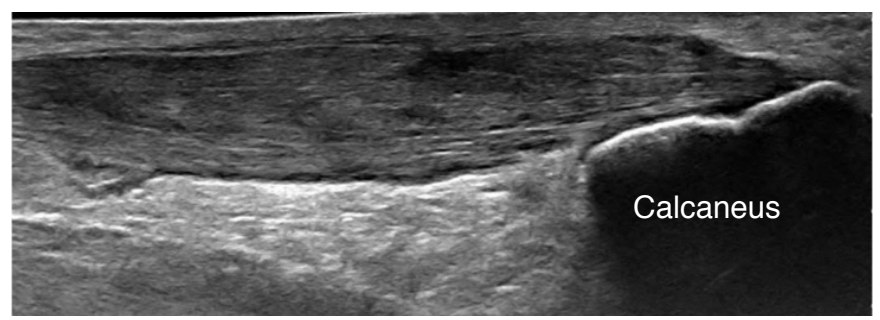

B

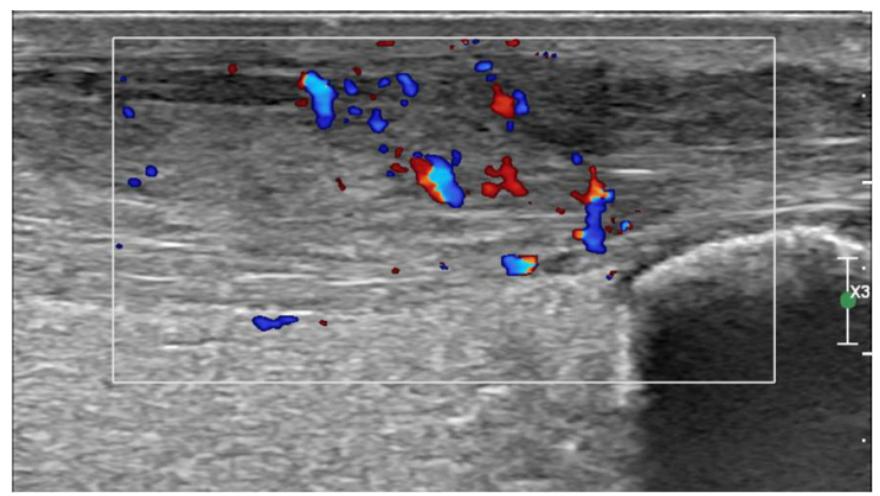

C

Fig. 10. Achilles tendinitis in a 63-year-old man.

A. A previous Tc-99m HDP whole body bone scan shows multifocal bone uptake in the right knee, left ankle, right third proximal interphalangeal joint, and the left first metacarpophalangeal joint, demonstrating rheumatoid arthritis. Several years later, the patient complained of right ankle swelling. B. Longitudinal scan of ultrasonography over the posterior ankle shows marked thickening and heterogeneous echogenicity of the right distal Achilles tendon. C. Color Doppler ultrasonography shows markedly increased color flow in the Achilles tendon. This is a case of Achilles tendinitis in a patient with rheumatoid arthritis. 


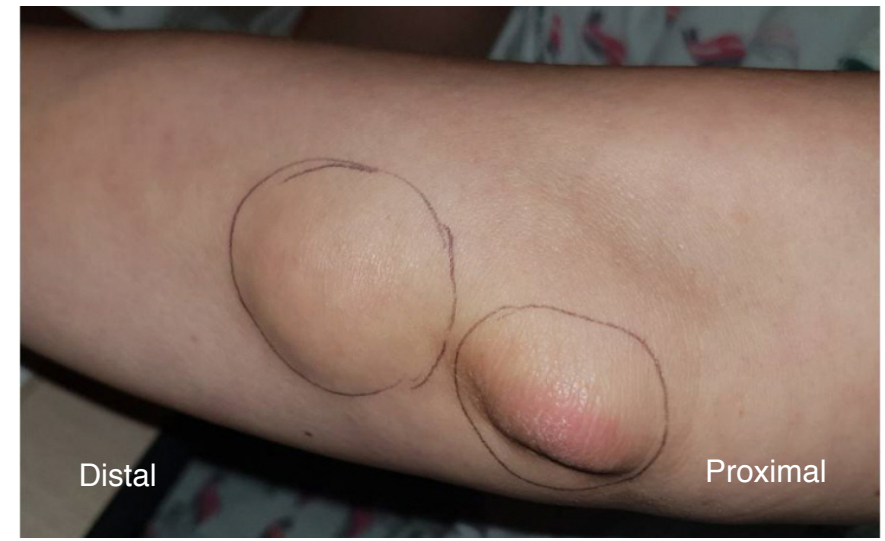

A

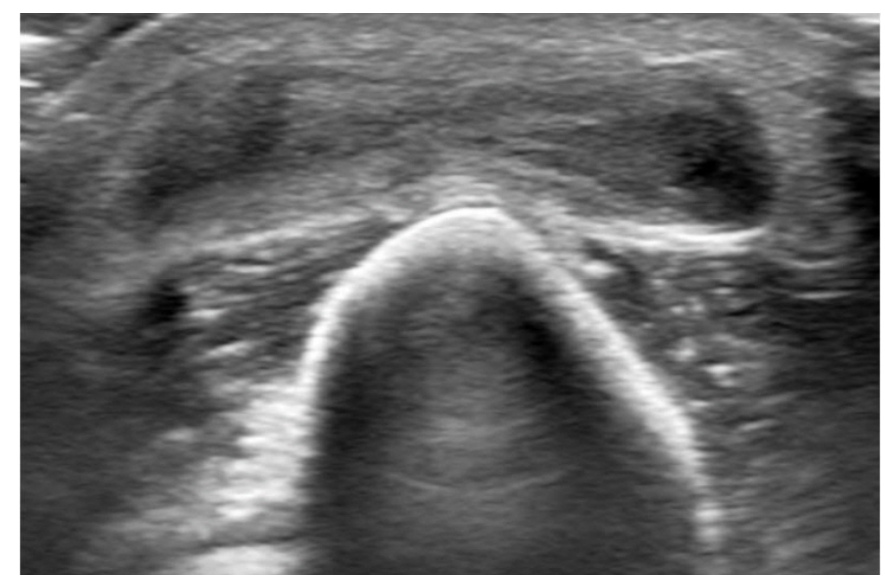

C

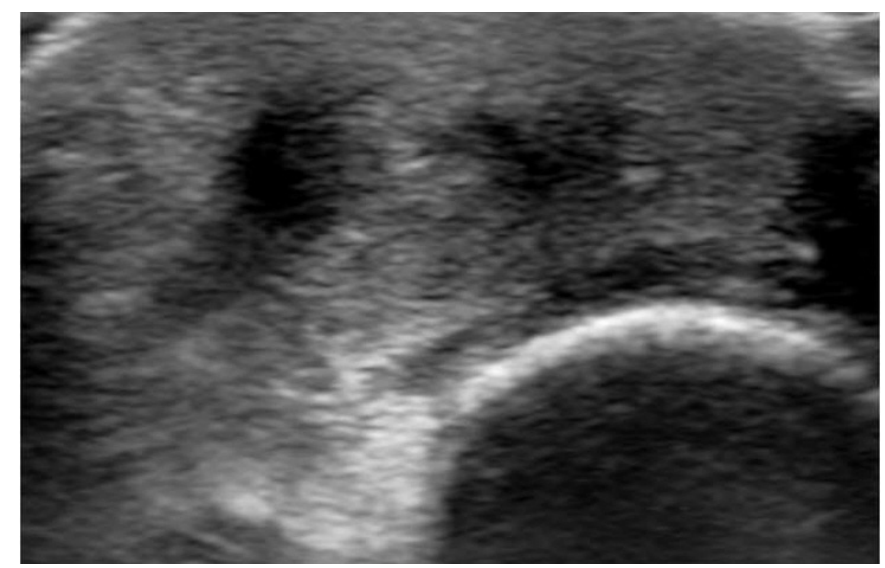

$\mathrm{E}$

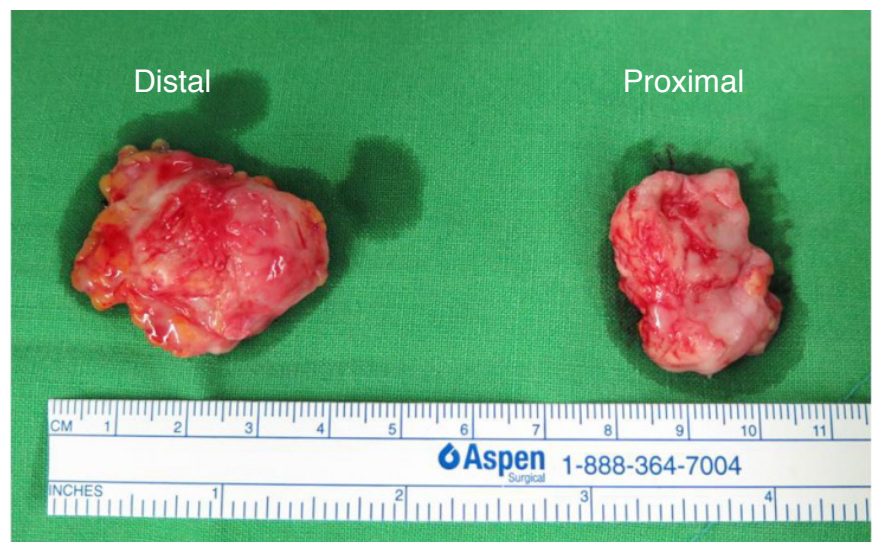

B

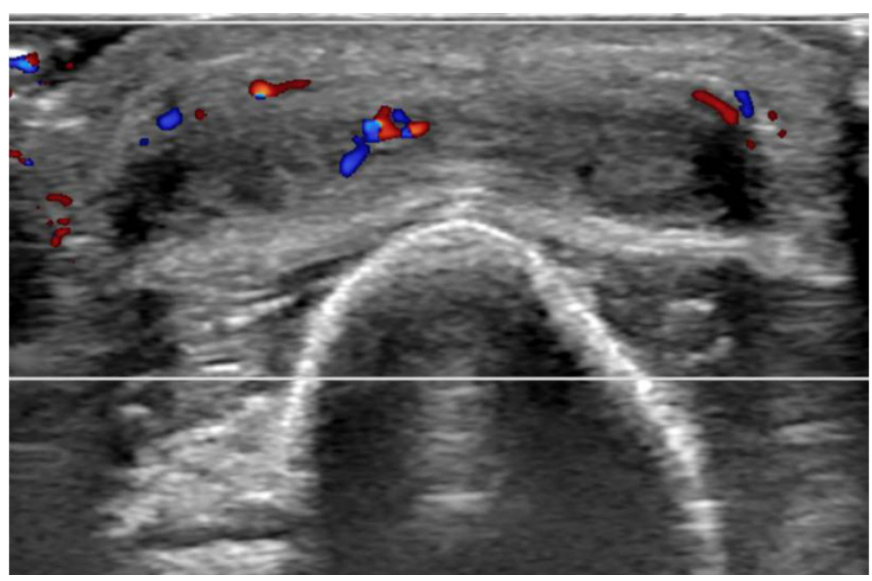

D

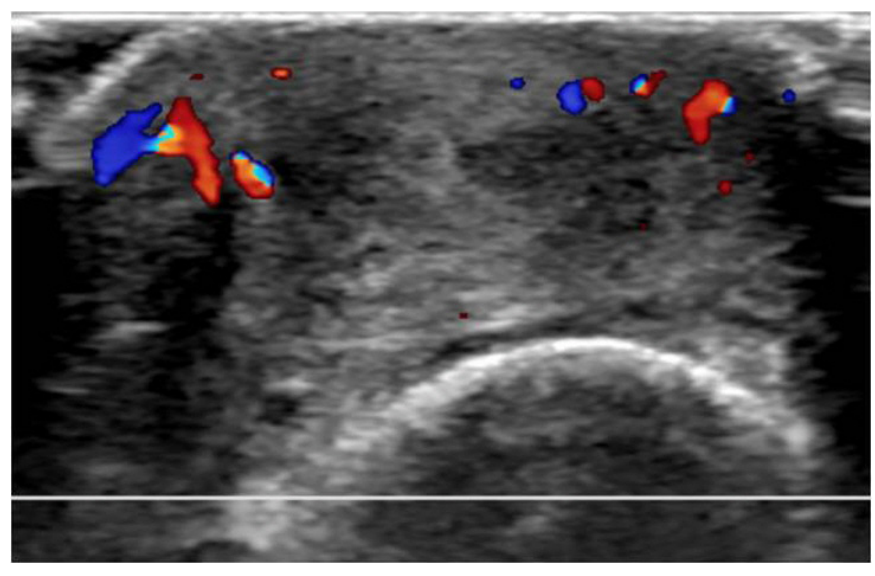

$\mathrm{F}$

Fig. 11. Olecranon bursitis accompanied by a rheumatoid nodule in the elbow in a 51-year-old woman.

A. A patient with seropositive rheumatoid arthritis had two palpable lesions for 3 months on the left elbow. The lesions were removed by surgical excision. B. On the pathology report, the proximal mass was confirmed as a rheumatoid nodule and the distal mass as olecranon bursitis. C. The distal lesion (olecranon bursitis) shows an oval, thick-walled subcutaneous lesion with a small amount of internal fluid collection. D. Hyperemia is shown on color Doppler ultrasonography. E. The proximal lesion, confirmed as a rheumatoid nodule, appears as an ill-defined, heterogeneous, and hypoechoic mass at the posterior aspect of the left elbow. F. Color Doppler ultrasonography shows increased vascularity of the mass. 


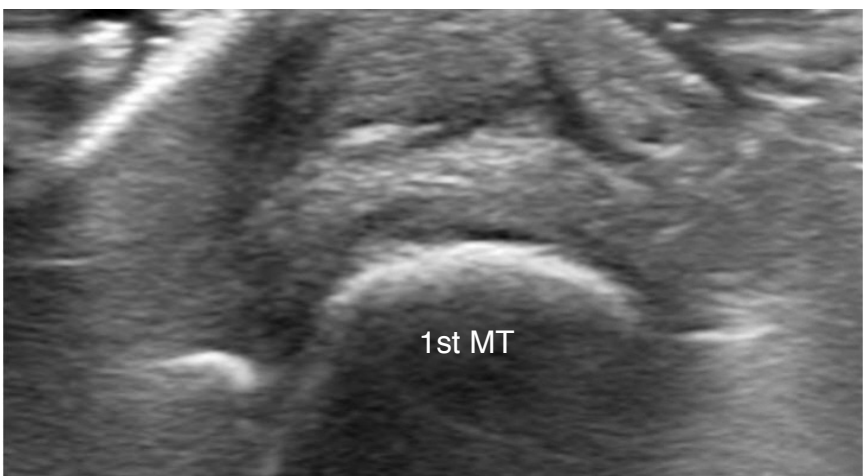

A

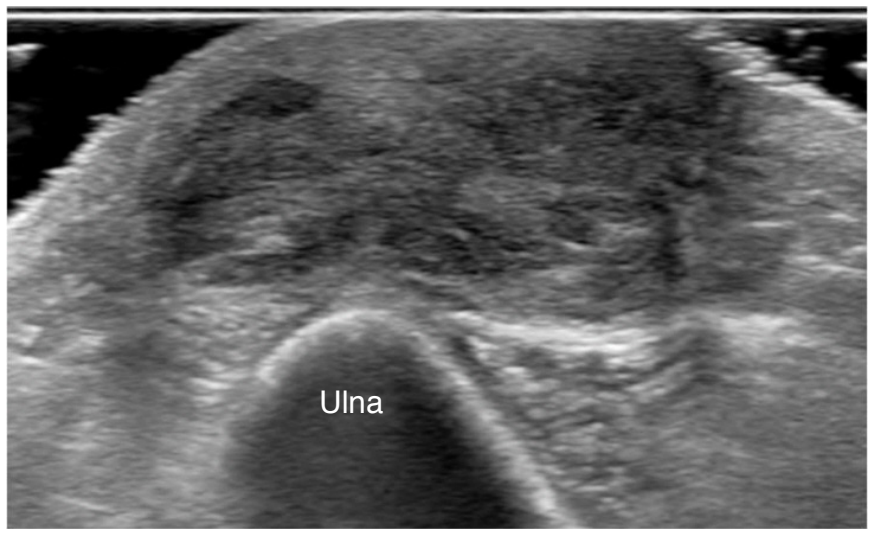

C

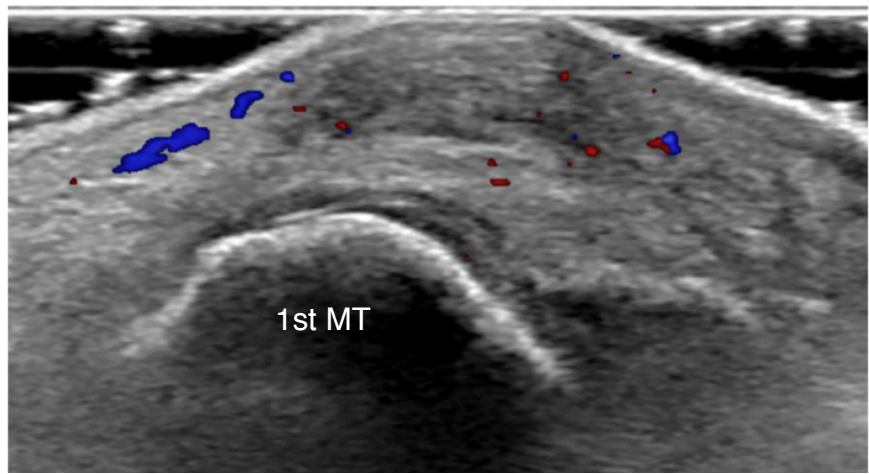

B

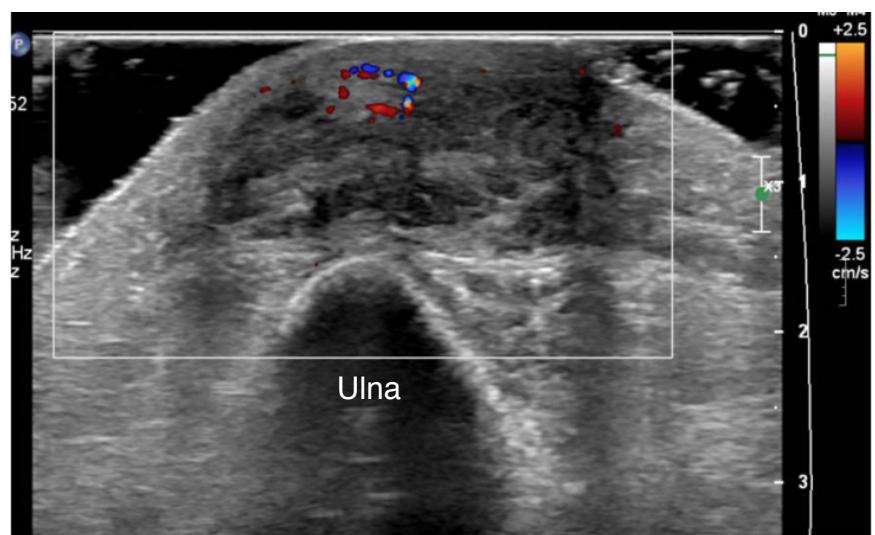

D

Fig. 12. Rheumatoid nodules at the left first metatarsophalangeal joint level and left elbow in a 56-year-old woman.

A patient on longstanding medication for seropositive rheumatoid arthritis had pain on the first metatarsophalangeal joint area in the left foot when standing and walking, and also in the left elbow. A. On grayscale ultrasonography, a 1.3-cm ovoid and hypoechoic mass is seen in the subcutaneous layer over the first metatarsal (MT) head. B. Increased vascularity of the lesion is noted on color Doppler ultrasonography. This is a case of a rheumatoid nodule in the left first metatarsophalangeal joint area. C. Another 3-cm heterogeneous hypoechoic subcutaneous mass overlies the posterior aspect of the left olecranon. D. It has increased vascularity on the color Doppler image. This is a case of a rheumatoid nodule at the left elbow.

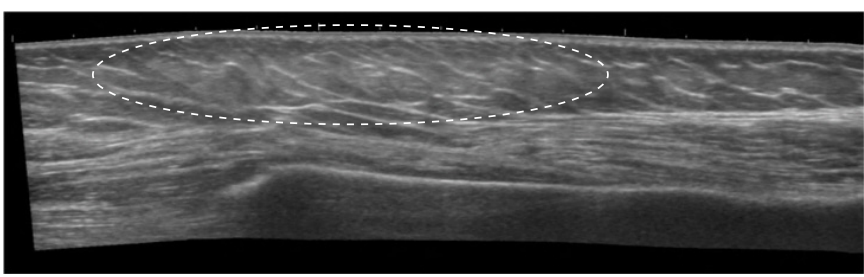

A

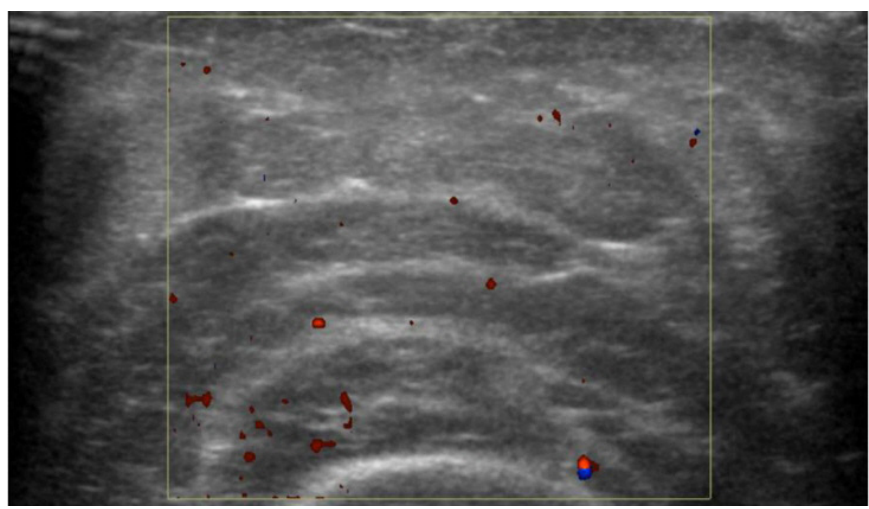

B

Fig. 13. Panniculitis in the left upper arm in a 66-year-old woman.

A patient with rheumatoid arthritis complained of pain in a soft-tissue lesion located at the left upper arm for 5 months. A. Ultrasonography over the lesion shows ill-defined and increased echogenicity of the subcutaneous fat layer of the left upper arm (dashed circle). B. Slightly increased vascularity on color Doppler ultrasonography is also seen. This case represents panniculitis in the subcutaneous fat layer in a patient with rheumatoid arthritis. 


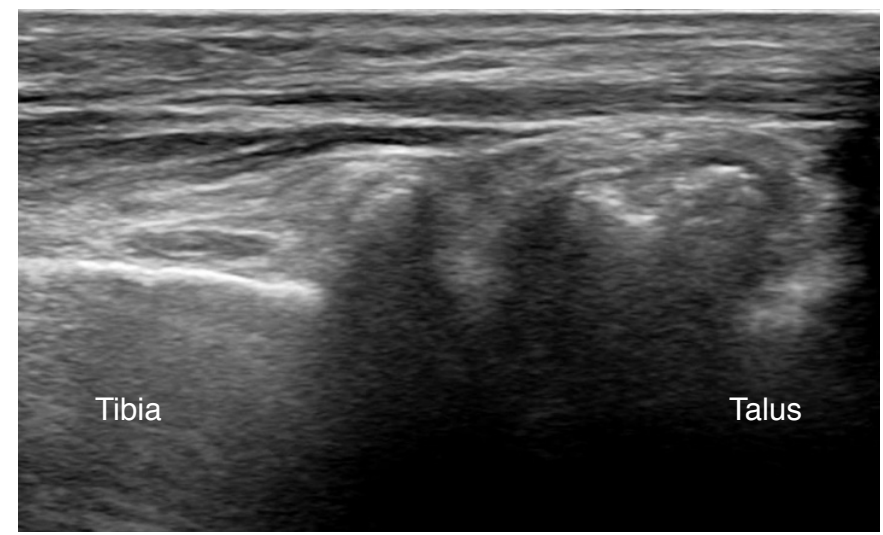

A

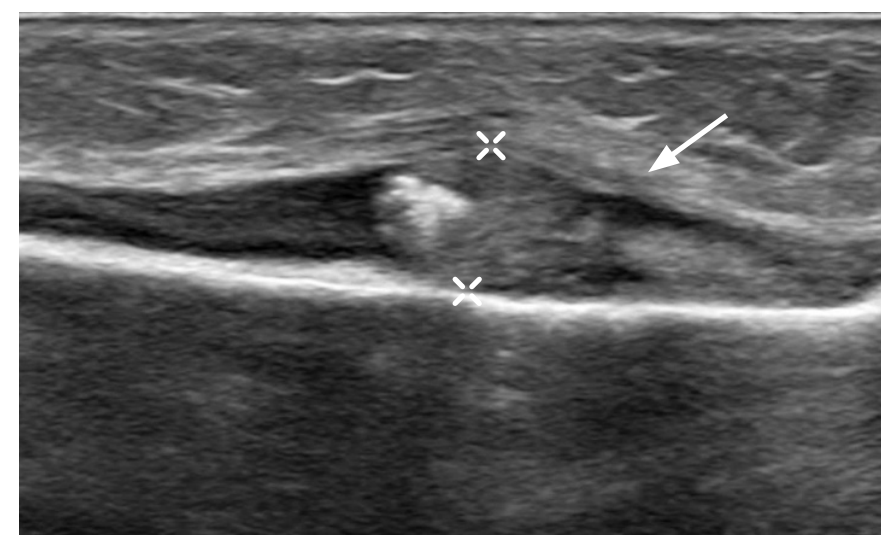

C

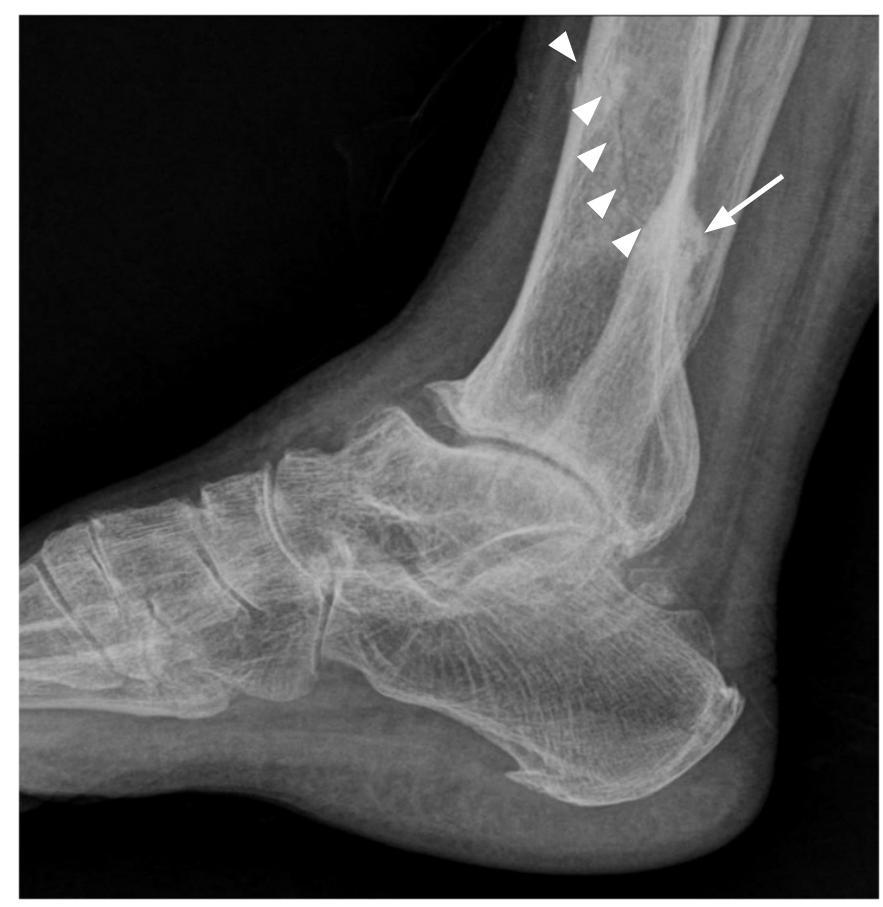

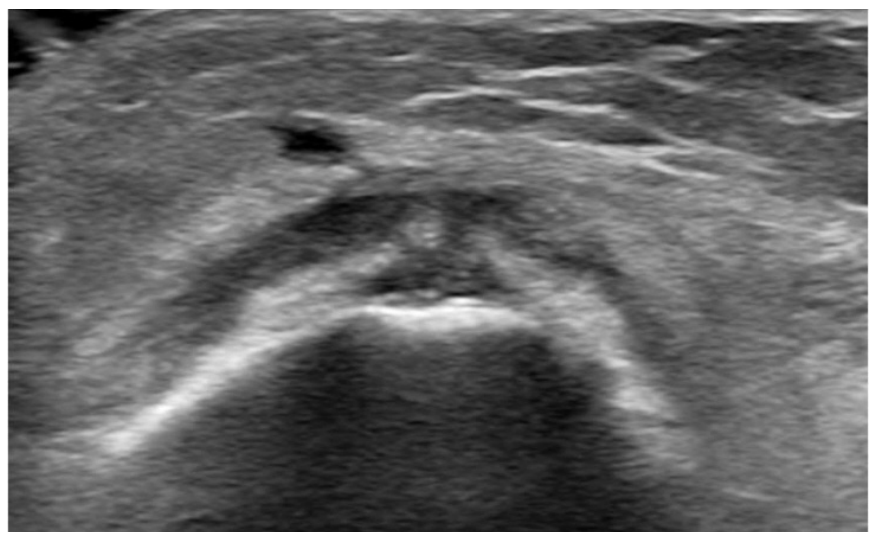

B

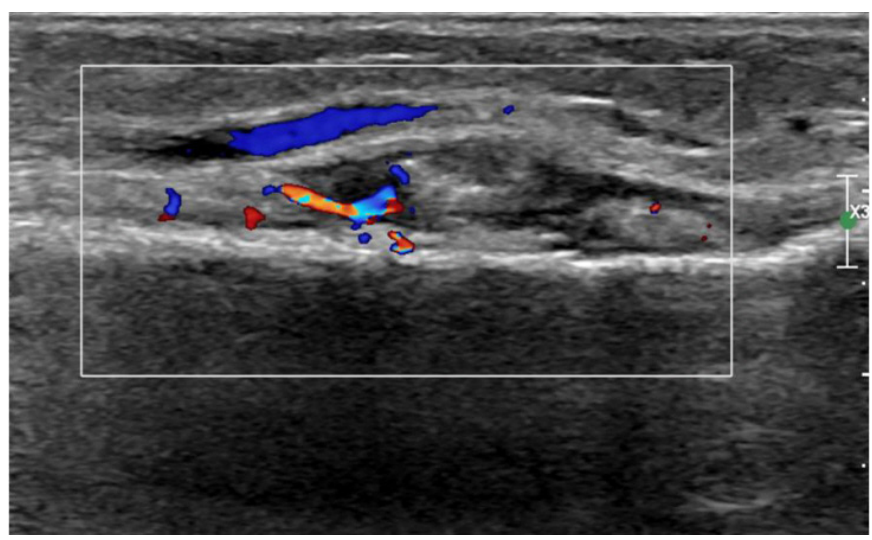

D

Fig. 14. Insufficiency fracture of the distal tibia in a 68-year-old woman.

A patient complained of swelling in the right ankle. She had been taking a corticosteroid and methotrexate for rheumatoid arthritis. A. On ultrasonography, there is no evidence of synovitis in the anterior recess of the right ankle. B. An axial scan over the right distal tibia shows periosteal thickening with surrounding soft-tissue swelling. C. A longitudinal scan shows an echogenic nodular lesion (arrow) on the medial aspect of the distal tibia. D. On color Doppler ultrasonography, there is hyperemia at the lesion. E. Subsequently, a plain radiograph reveals diffuse osteopenia and an oblique fracture line (arrowheads) with callus formation (arrow) on the right distal tibia, suggesting stress fracture. The stress fracture may have been due to the longstanding use of a corticosteroid and methotrexate. 


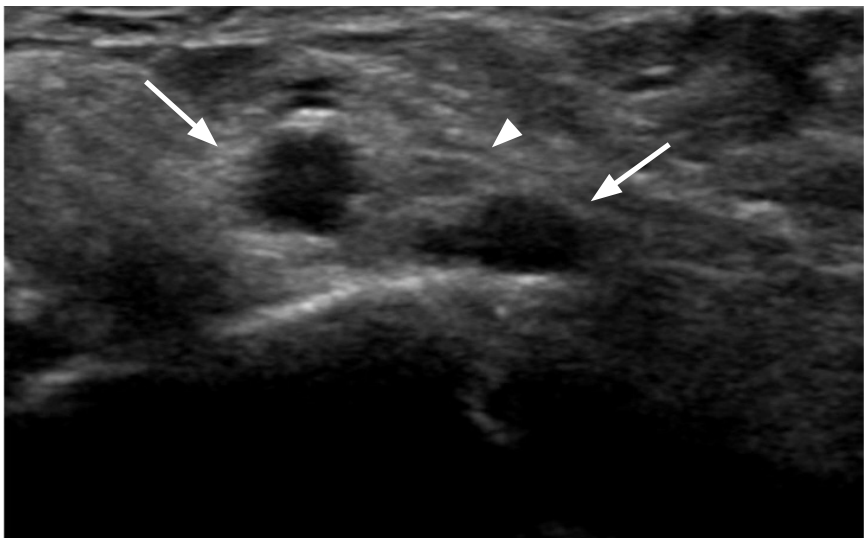

A

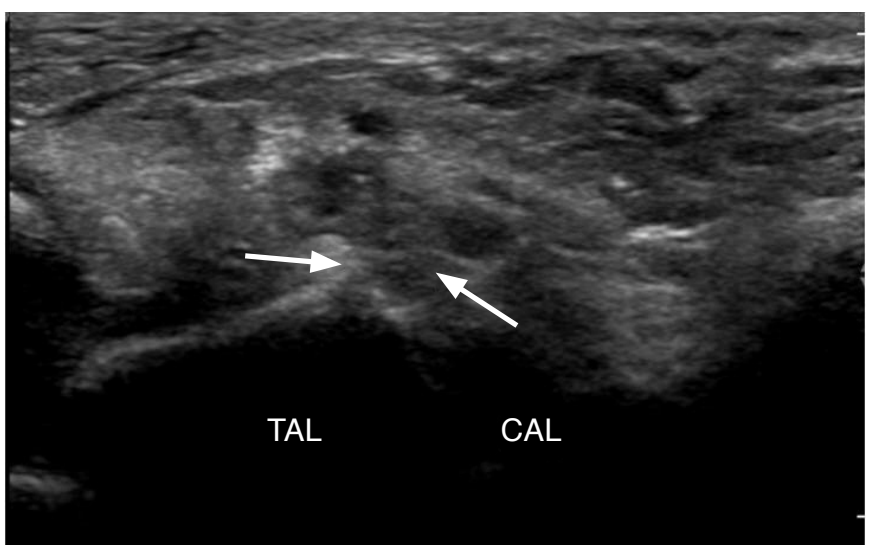

C

\section{Bone}

The inflammatory nature of RA increases the risk of bone loss and fracture. Moreover, corticosteroid and methotrexate usage renders the bone more vulnerable to fracture. Thus, the prevalence of insufficiency fracture is higher in patients with RA $(0.8 \%)$, and it can cause significant morbidity [17]. Diagnosing insufficiency fracture is challenging because it frequently masquerades as an inflammatory disease exacerbation. Moreover, the diagnosis of insufficiency fracture may be delayed by the late appearance of a callus; radiographs are normal until periosteal reactions appear or until cortical thickening at 2-4 weeks after symptom onset. Therefore, radiologists should suspect the presence of an insufficiency fracture if there is a mismatch between clinical symptoms and the radiologic findings of other structures associated with RA, particularly in highrisk patients.

Magnetic resonance imaging and bone scintigraphy are gold standards for the early diagnosis of insufficiency fractures. Although it is generally not the method of choice, US also can be useful for the early detection of insufficiency fracture with good sensitivity and specificity [18]. The following US findings are highly suggestive

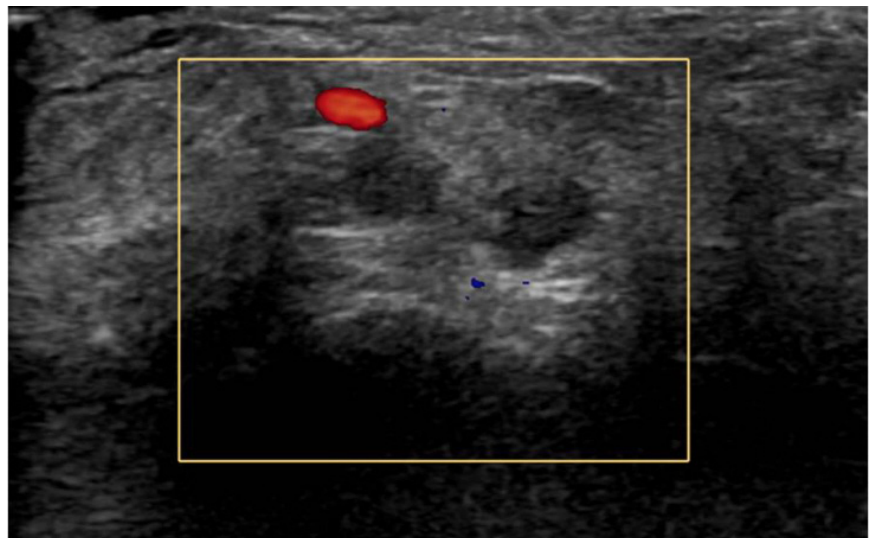

B

Fig. 15. Tarsal tunnel syndrome in a 76-year-old woman.

A patient with seropositive rheumatoid arthritis for more than 10 years complained of pain and swelling in the right ankle. A. On grayscale ultrasonography, there are anechoic lesions (arrows) in the tarsal tunnel, at the medial talocalcaneal joint level, compressing the tibial nerve (arrowhead). B. On color Doppler ultrasonography, the tibialis posterior artery has color flow, but the anechoic lesion does not have color flow, indicating that these are multiseptated cysts. C. The cystic lesions extend from the medial talocalcaneal joint (arrows). This is a case of ganglion cysts from the talocalcaneal joint causing tarsal tunnel syndrome.

of insufficiency fracture: hypoechoic periosteal elevation above the cortical bone, increased posterior shadowing, fluid collection surrounding an injured bone, and increased vascularity on color Doppler (Fig. 14) [19].

\section{Nerve}

RA is a systemic inflammatory disease with potential neurologic manifestations. The clinical presentations of peripheral neuropathy include entrapment neuropathy (carpal, cubital, and tarsal tunnel syndrome, and peroneal neuropathy), distal sensory neuropathy, combined sensorimotor polyneuropathy, and mononeuritis multiplex. Entrapment neuropathies are the most common cause of neurologic impairment [8] and may occur at sites where nerves pass. Joint deformities, as well as inflamed synovium, ligaments, and tendon sheaths, may compress the peripheral nerves close to the joints or bursae. US can distinguish entrapment neuropathies by detecting the anatomical site and structures compressing the nerve. For instance, the inflammatory conditions of RA with tendon swelling, synovitis, effusion, or extra-articular ganglion displacing the tibial nerve can be visualized on US for tarsal tunnel syndrome 


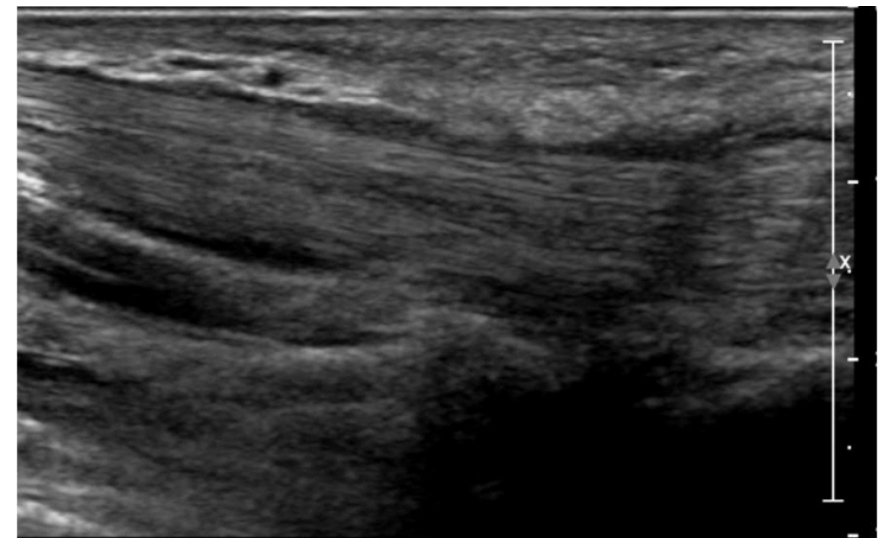

A

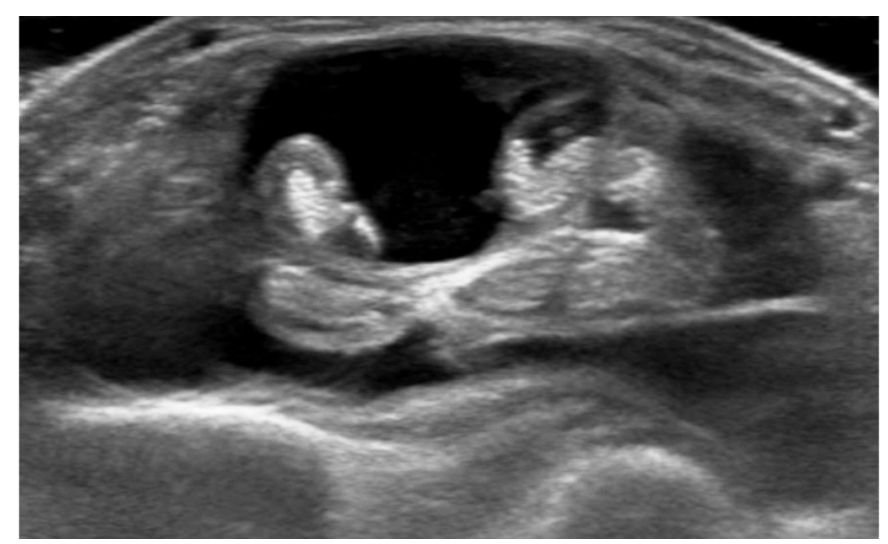

C

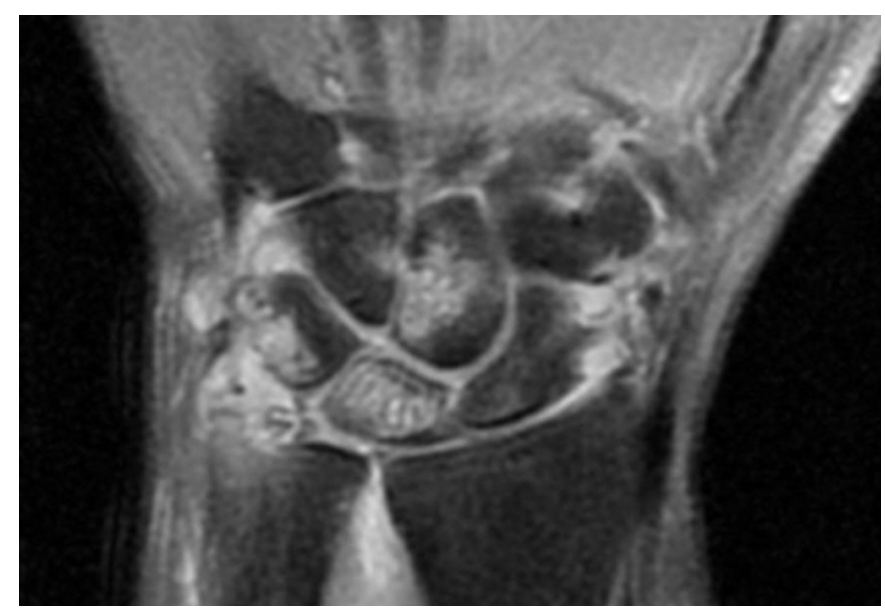

$\mathrm{E}$

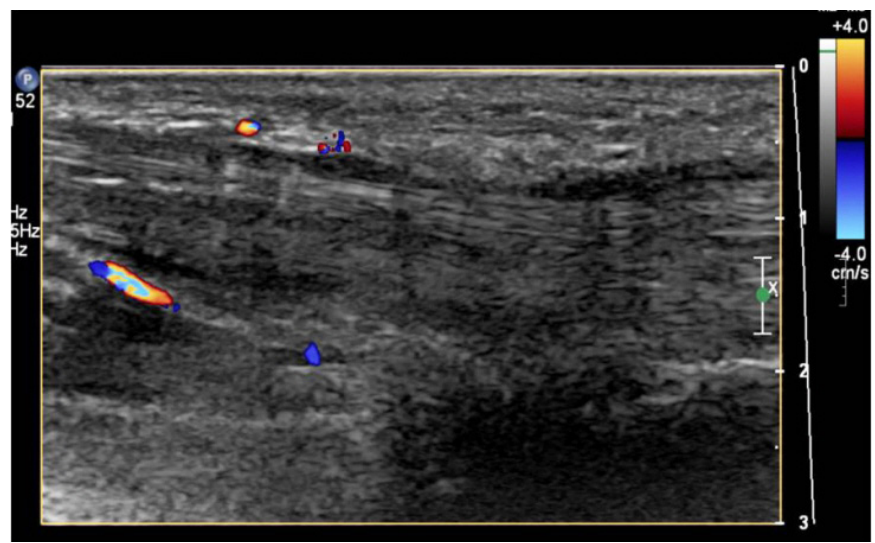

B

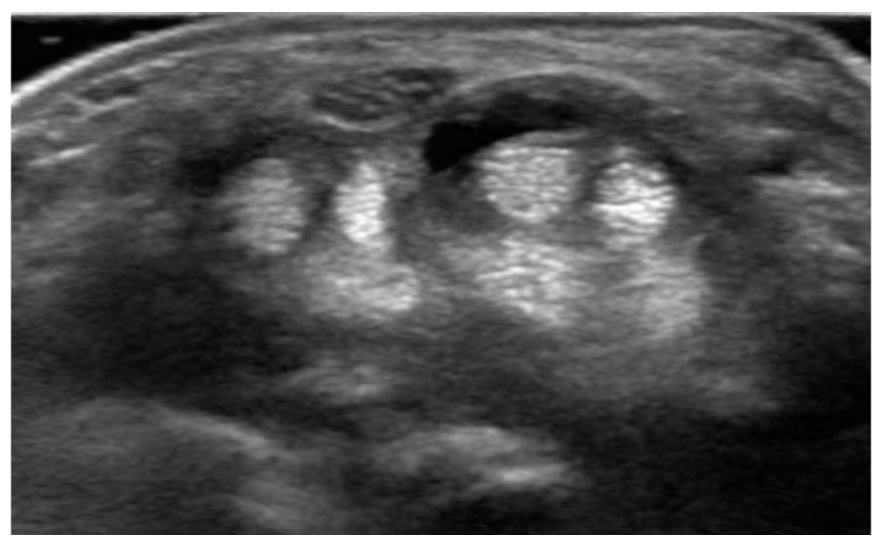

D

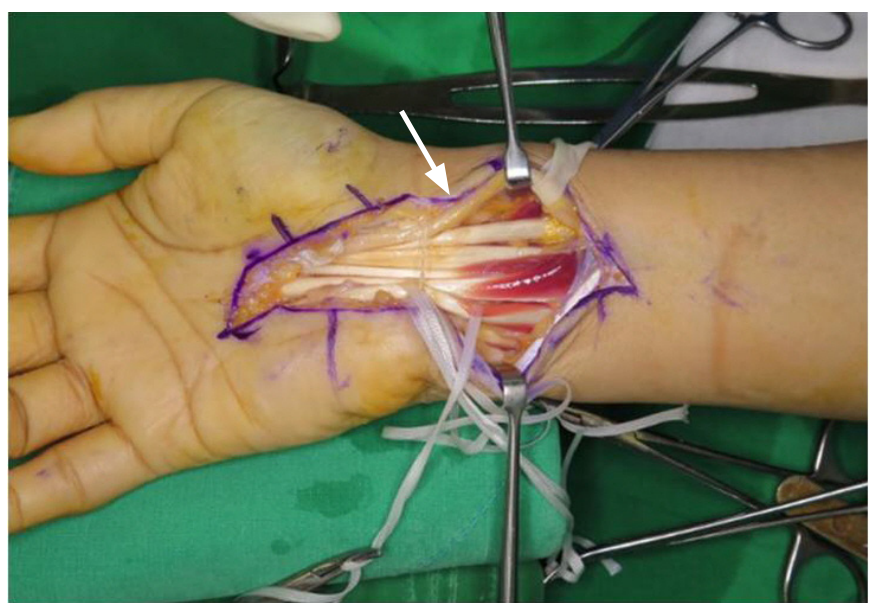

$\mathrm{F}$

Fig. 16. Carpal tunnel syndrome in a 50-year-old woman.

A patient who had been on longstanding medication for rheumatoid arthritis complained of right wrist pain and right-hand numbness. A. On a longitudinal scan of ultrasonography, diffuse swelling of flexor digitorum tendons with thickened tendon sheaths is seen. B. Increased vascularity of the lesion is noted on color Doppler ultrasonography. C. Fluid collection is noted over the common flexor tendon sheath, at the distal forearm level. D. The fluid collection extends to the carpal tunnel and the median nerve is compressed by swollen tendons and the fluid collection at the carpal tunnel level. E. Multifocal peri-articular bone erosions are shown in the carpal bone on the coronal scan of magnetic resonance imaging (STIR image). Eventually, surgical decompression was done. F. Carpal tunnel is released by synovectomy and the median nerve is exposed on gross photography (arrow). This is a case of carpal tunnel syndrome induced by rheumatoid arthritis. 
(Fig. 15) [20]. Carpal tunnel syndrome is the most common entrapment neuropathy, and RA is the most commonly associated rheumatologic disorder [21]. Smerilli et al. [21] reported that the sonographic spectrum of carpal tunnel syndrome in patients with RA is characterized by an inflammatory pattern, which is defined by finger flexor tendon tenosynovitis and/or radio-carpal joint synovitis, with marked median nerve swelling being the dominant feature of idiopathic carpal tunnel syndrome (Fig. 16).

\section{Conclusion}

A better understanding of the broad spectrum of RA, including its peri- and extra-articular manifestations, could improve the accuracy of RA diagnoses and assist rheumatologists in making more appropriate therapeutic decisions. US is a useful, relatively inexpensive, and non-invasive modality to detect various RA manifestations.

ORCID: Ji Young Suh: https://orcid.org/0000-0003-1731-181X; Sun-Young Park: https://orcid.org/0000-0001-6500-3411; Sung Hye Koh: https://orcid.org/0000-00024293-5889; In Jae Lee: https://orcid.org/0000-0001-9775-5677; Kwanseop Lee: https://orcid.org/0000-0002-8951-4512

\section{Author Contributions}

Conceptualization: Suh JY, Park SY, Koh SH. Data acquisition: Park SY, Koh SH. Data analysis or interpretation: Suh JY, Park SY, Koh SH. Drafting of the manuscript: Suh JY, Park SY. Critical revision of the manuscript: Koh SH, Lee IJ, Lee K. Approval of the final version of the manuscript: all authors

\section{Conflict of Interest}

No potential conflict of interest relevant to this article was reported.

\section{References}

1. Resnick DL, Kransdorf MJ. Bone and joint imaging. Philadelphia, PA: Elsevier Health Sciences, 2004.

2. Janta I, Stanciu D, Hinojosa M, Nieto-Gonzalez JC, Valor L, Bello $\mathrm{N}$, et al. Structural damage in rheumatoid arthritis: comparison between tendon damage evaluated by ultrasound and radiographic damage. Rheumatology (Oxford) 2016;55:1042-1046.

3. Filippucci E, Gabba A, Di Geso L, Girolimetti R, Salaffi F, Grassi W. Hand tendon involvement in rheumatoid arthritis: an ultrasound study. Semin Arthritis Rheum 2012;41:752-760.

4. Sudol-Szopinska I, Jans L, Teh J. Rheumatoid arthritis: what do MRI and ultrasound show. J Ultrason 2017;17:5-16.

5. Rizzo C, Ceccarelli F, Gattamelata A, Vavala C, Valesini G, lagnocco
A. Ultrasound in rheumatoid arthritis. Med Ultrason 2013;15:199208.

6. Plotkin B, Sampath SC, Sampath SC, Motamedi K. MR imaging and US of the wrist tendons. Radiographics 2016;36:1688-1700.

7. Vyas S, Bhalla AS, Ranjan P, Kumar S, Kumar U, Gupta AK. Rheumatoid arthritis revisited: advanced imaging review. Pol J Radiol 2016;81:629-635.

8. Khurana R, Berney SM. Clinical aspects of rheumatoid arthritis. Pathophysiology 2005;12:153-165.

9. De Backer Al, Mortele KJ, Vanhoenacker FM, Parizel PM. Imaging of extraspinal musculoskeletal tuberculosis. Eur J Radiol 2006;57:119130.

10. Kim HA, Kim SH, Seo Yl. Ultrasonographic findings of the shoulder in patients with rheumatoid arthritis and comparison with physical examination. J Korean Med Sci 2007;22:660-666.

11. Bowen CJ, Hooper L, Culliford D, Dewbury K, Sampson M, Burridge $J$, et al. Assessment of the natural history of forefoot bursae using ultrasonography in patients with rheumatoid arthritis: a twelvemonth investigation. Arthritis Care Res (Hoboken) 2010;62:17561762.

12. McGonagle D, Lories RJ, Tan AL, Benjamin M. The concept of a "synovio-entheseal complex" and its implications for understanding joint inflammation and damage in psoriatic arthritis and beyond. Arthritis Rheum 2007;56:2482-2491.

13. Suzuki T, Okamoto A. Ultrasound examination of symptomatic ankles in shorter-duration rheumatoid arthritis patients often reveals tenosynovitis. Clin Exp Rheumatol 2013;31:281-284.

14. Suzuki T, Hidaka Y, Seri Y. Retrocalcaneal bursitis precedes or accompanies achilles tendon enthesitis in the early phase of rheumatoid arthritis. Clin Med Insights Arthritis Musculoskelet Disord 2018;11:1179544118781094.

15. Nalbant S, Corominas H, Hsu B, Chen LX, Schumacher HR, Kitumnuaypong $\mathrm{T}$. Ultrasonography for assessment of subcutaneous nodules. J Rheumatol 2003;30:1191-1195.

16. Rodriguez-Jimenez $P$, Chicharro P, Reolid A, Munoz-Aceituno E, Fraga J, Llamas-Velasco M. Sequential appearance of small vessel vasculitis and neutrophilic panniculitis in a patient with rheumatoid arthritis: further insight into the mechanism of this infrequent panniculitis. Int J Dermatol 2019;58:e198-e200.

17. Dreher R, Buttgereit F, Demary W, Gortz B, Hein G, Kern P, et al. Insufficiency fractures in rheumatology. Case report and overview. $Z$ Rheumatol 2006;65:417-423.

18. Banal F, Gandjbakhch F, Foltz V, Goldcher A, Etchepare F, Rozenberg $S$, et al. Sensitivity and specificity of ultrasonography in early diagnosis of metatarsal bone stress fractures: a pilot study of 37 patients. J Rheumatol 2009;36:1715-1719.

19. Bodner G, Stockl B, Fierlinger A, Schocke M, Bernathova M. Sonographic findings in stress fractures of the lower limb: preliminary findings. Eur Radiol 2005;15:356-359. 
20. Ibrahim IK, Medani SH, El-Hameed MM, Imam MH, Shaaban MM. Tarsal tunnel syndrome in patients with rheumatoid arthritis, electrophysiological and ultrasound study. Alexandria J Med 2013;49:95-104.
21. Smerilli G, Di Matteo A, Cipolletta E, Carloni S, Incorvaia A, Di Carlo $M$, et al. Ultrasound assessment of carpal tunnel in rheumatoid arthritis and idiopathic carpal tunnel syndrome. Clin Rheumatol 2021;40:1085-1092. 